\title{
Unified scaling of the structure and loading of nanoparticles formed via diffusion-limited coalescence
}

\author{
Teresita Rode García ${ }^{1}$, Araceli García Ac ${ }^{1}$, Augustine Lalloz ${ }^{1}$, Francois-Xavier Lacasse ${ }^{1}$, Patrice \\ Hildgen ${ }^{1}$, Jean-Michel Rabanel ${ }^{1, \$,}$, Xavier Banquy ${ }^{1, *}$ \\ ${ }^{1}$ Faculté de Pharmacie, Université de Montréal, C.P. 6128, Succursale Centre-ville, Montréal, \\ Québec H3C 3J7, Canada. \\ ${ }^{\$}$ Present address: Centre INRS Institut Armand-Frappier, 531 Boulevard des Prairies, Laval, \\ Québec H7V 1B7, Canada \\ *Corresponding authors: xavier.banquy@umontreal.ca; jean-michel.rabanel@iaf.inrs.ca
}

Keywords: Polymeric Nanoparticles, nanoprecipitation, micromixing, drug loading, polylactic acid, diblock polymers, polyethylene glycol.

\section{Abstract}

The present study establishes the scaling laws describing the structure of spherical nanoparticles formed via diffusion-limited coalescence. We produced drug loaded nanoparticles from a poly(ethylene glycol)-poly(D,L-lactic acid) diblock polymer (PEG-b-PLA) using the nanoprecipitation method using different types of micromixing chambers in order to explore multiple mixing regimes and characteristic times. We first show that the drug loading of the nanoparticles is not controlled by the mixing time but solely by the drug to polymer ratio in the feed (D:P) and the hydrophobicity of the drug scaled via the partition coefficient $\mathrm{P}$. We then procure compelling evidence that particles formed via diffusion/coalescence exhibit a relative distribution of PEG blocks between the particle core and its shell that depends only on mixing conditions (not on D:P). Scaling laws of PEG relative distribution and chain surface density were derived in different mixing regimes and showed excellent agreement with experimental data. In particular, results made evident that PEG blocks entrapment in the core of the particles occurs in the slow mixing regime and favors the overloading (above the thermodynamic limit) of the particles with hydrophilic drugs. The present analysis compiles effective guidelines for the scale up of nanoparticles structure and properties with mixing conditions which should facilitate their future translation to medical and industrial settings. 
2 Graphical abstract
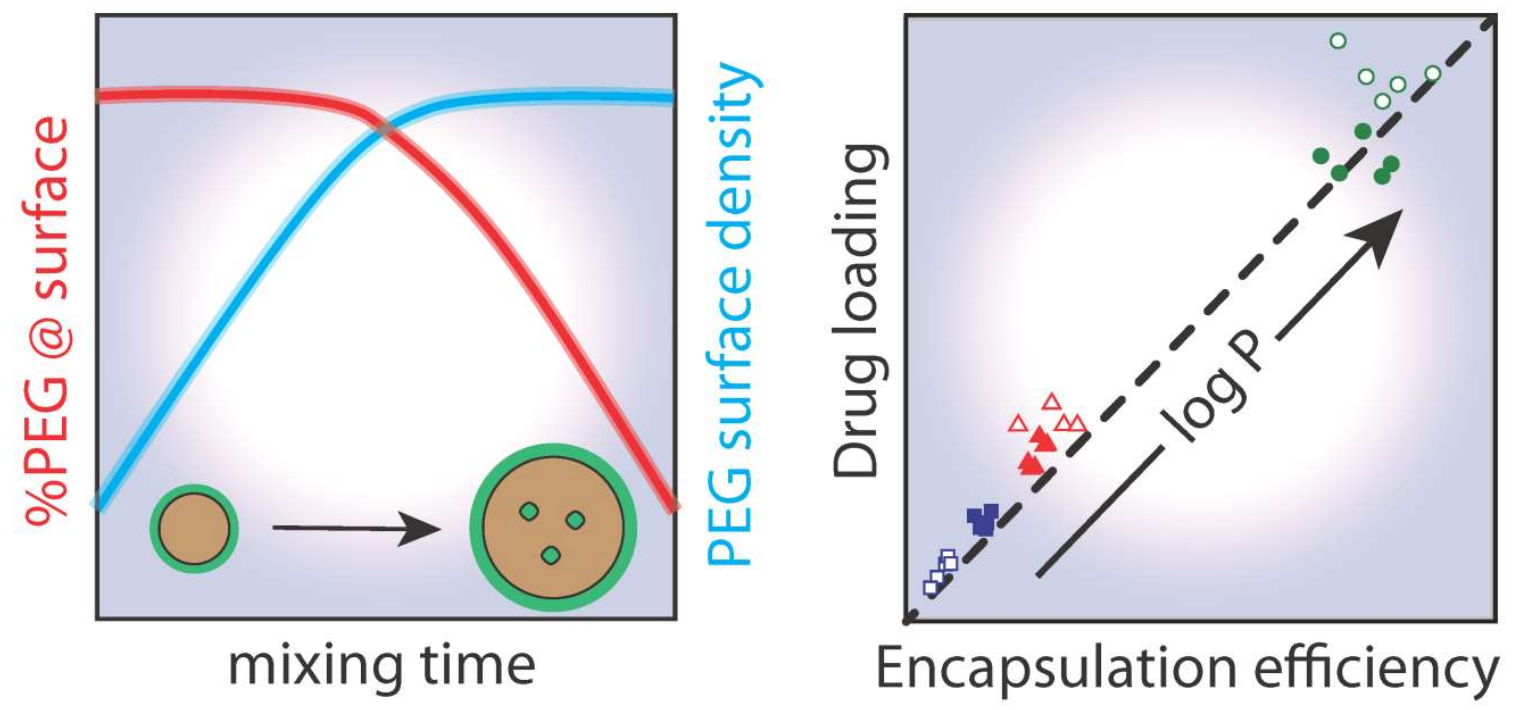

4 


\section{Introduction}

2 Encapsulation of drugs in polymeric nanoparticles (NPs) represents a successful strategy to

3 ameliorate bioavailability and stability of therapeutic agents, as well as to improve controlled drug

4 release and drug targeting ${ }^{1-2}{ }^{3-5}$. These advantages are highly related to the physicochemical

5 properties of the NPs.:Size ${ }^{6-7}{ }^{8-9}$, Zeta Potential ${ }^{3,8,10-11}$, NP surface composition ${ }^{12-14}$ and drug

$6 \quad$ loading ${ }^{15-17} 18-19$.

7 In spite of the great advantages of nanoformulations to deliver drugs, the methods used to prepare

8 them are still in development and fundamental understanding of the multiple phenomena leading to

9 their formation is still lacking. Frequently, NPs properties tend to vary drastically during fabrication

10 scale-up ${ }^{20}$. Drift in NPs properties influences the performance of the formulated drug in vivo and

11 represent one of the many issues encountered during clinical trials. Therefore, it is paramount to

12 identify and control all the process variables that may influence the physicochemical properties of

13 particulate nanoformulations.

14 There are several methods for the preparation of polymeric "core-shell" NPs ${ }^{21}$ which have been

15 classified in two-step and one-step procedures. Typically, two-step methods involve the preparation

16 of nanodroplets through the formation of an o/w emulsion, followed by the removal of the organic

17 solvent ${ }^{22}$. On the other hand, one-step methods are based on the spontaneous precipitation of the

18 polymer in solution through solvent displacement (also called ouzo effect). ${ }^{23-24}$ Nanoprecipitation

19 methods have many advantages over other fabrication techniques: simplicity, scalability, good

20 batch to batch reproducibility, avoidance of toxic solvents as well as low energy input and good

21 particle size control. ${ }^{25-26}$

22 Nanoprecipitation can be performed in a batch mixer or continuously. In that last modality, several

23 processes have been developed using micromixing devices. ${ }^{27-30}$ The Staggered Herringbone

24 Micromixer (SHM) ${ }^{29-30}$ and the confined impinging jet micromixer $(\mathrm{CIJM})^{27}$ are two of the

25 numerous devices developed so far in this exponentially growing field. Both methods offer robust

26 control over the fabrication process and over the properties of the produced NPs, compared with the 
1 batch nanoprecipitation (BN) process (see Figure 1). Systematic comparison of these methods is

2 currently lacking generates uncertainty when the most appropriate method to prepare polymeric

3 NPs is to be chosen.

4 Regarding the nanoprecipitation method, it is well documented that the mixing speed or 5 characteristic time influence the formation of the NPs, their size and size distribution but the 6 rationalization of the observed trends so far has only emerged recently. ${ }^{24,27,31-32}$ Other structural

7 parameters such as core-shell structure or composition homogeneity are still largely unexplored and 8 require particular attention.

9 The aim of the present study was thus to prepare NPs by three different mixing processes, namely 10 Batch Nanoprecipitation (BN), Flash Micromixer Nanoprecipitation (FMN) and the Staggered 11 Herringbone Micromixer Nanoprecipitation (SHMN) which cover a large range of mixing 12 conditions and to determine the size, size polydispersity (PdI), and distribution of PEG blocks at the 13 NPs surface and in their core. Active compounds with different octanol/water partition coefficients 14 values, $\log \mathrm{P}$ (Table 1) were encapsulated and the encapsulation efficiency and the drug loading 15 were evaluated according to the fabrication method. The data generated allowed to establish scaling 16 laws of the NP structure and drug loading for different mixing regimes and to rationalize the 17 differences between each fabrication processes. The results of this study should contribute to 18 rationally correlate the properties of nanoformulations prepared from diblock material to their 19 fabrication process and facilitate their translation to large production settings. 


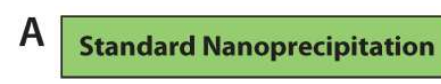

Batch Nanoprecipitation

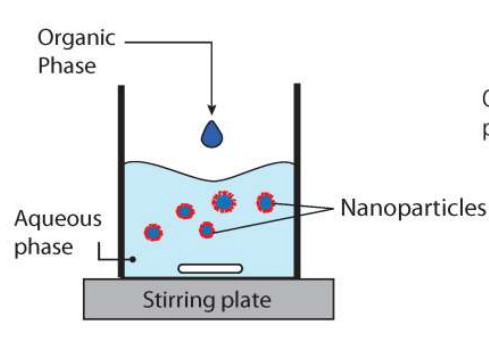

B
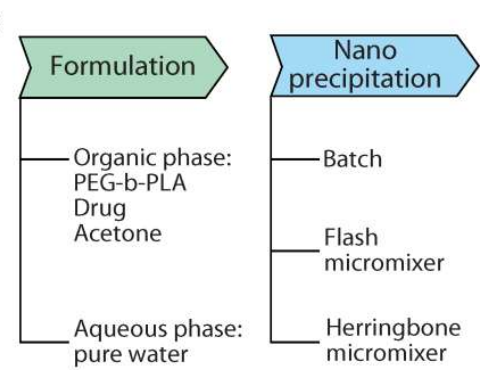
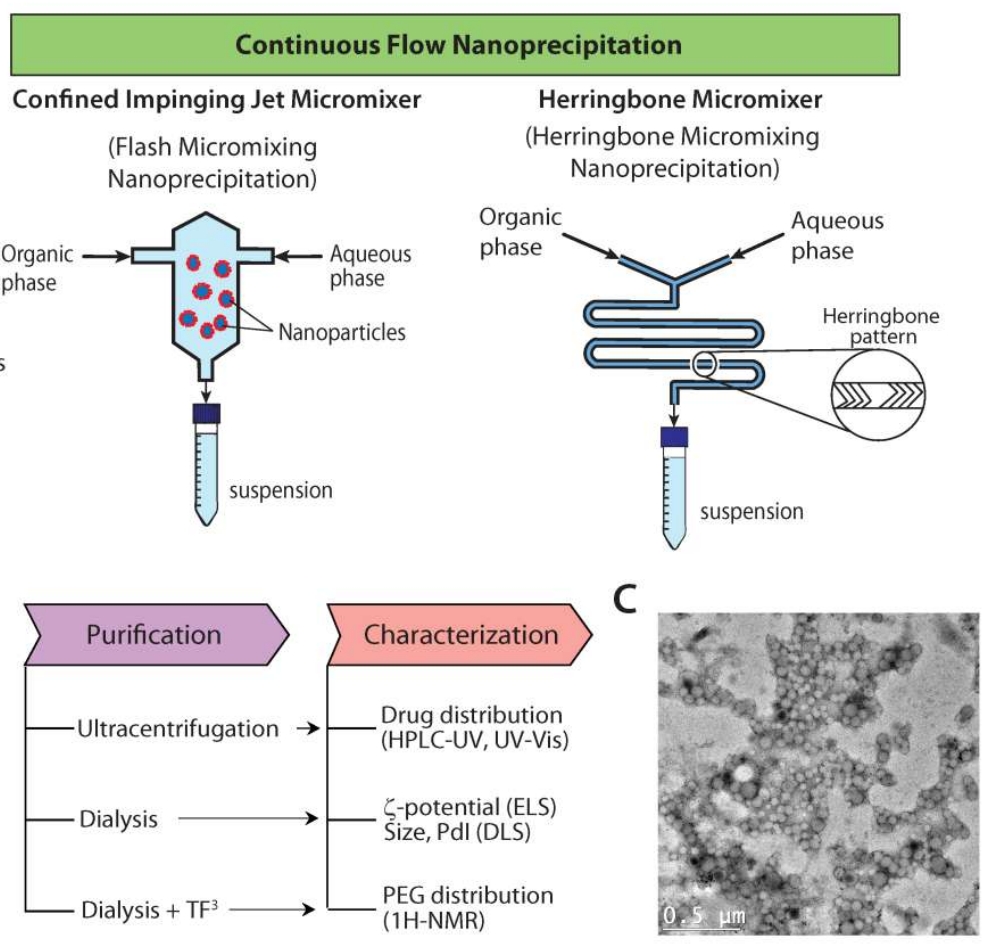

Figure 1. (A) Schematic representation of the different fabrication processes used in the present study. (B) Workflow diagram used to produce, purify and characterize the NPs. (C) Representative TEM image of NPs fabricated from PEG- $b$-PLA using CIJM.

\section{Experimental Section}

\section{Materials}

3,6 -Dimethyl-1,4-dioxane-2,5-dione, Tin(II) 2-ethylhexanoate and poly(ethylene glycol) $\left(\mathrm{M}_{\mathrm{W}}=\right.$ $2000 \mathrm{~g} / \mathrm{mol}$ ), ketoprofen ( $\geq 99 \%$ ), theophylline ( $\geq 99 \%$ ), cholecalciferol ( $>98 \%$ ), chloroform-d and deuterium oxide (1\% w/w 3-(trimehylsilyl)-1-proponesulfonic acid) were provided by Sigma Aldrich (Oakville, ON, Canada) and used without further purification. Acetonitrile (HPLC-grade), methanol (HPLC-grade) glacial acetic acid, acetone, toluene, tetrahydrofuran, and dichloromethane were obtained from Fisher Scientific (Ottawa, ON, Canada). 
1 Table 1. Physicochemical properties of the model drugs.

\begin{tabular}{ccccc}
\hline & Theophylline & Ketoprofen & Reference \\
\hline Molar mass $(\mathrm{g} / \mathrm{mol})$ & 180.164 & 254.281 & 384.64 & \\
$\log \mathrm{P}$ & 0 & 3.16 & -6.4 & {$\left[{ }^{33-34}\right]$} \\
Intrinsic $\log (\mathrm{S})$ & -1.38 & -3.33 & 0.00038 & {$\left[{ }^{37-38}\right]$} \\
Solubility $(\mathrm{mg} / \mathrm{mL})$ & 8.33 & 0.0213 & - & {$\left[{ }^{37}\right]$} \\
$\mathrm{pKa}$ & 8.81 & 4.12 & & \\
\hline
\end{tabular}

3 Synthesis of PEG-b-PLA polymer.

4 The synthesis of polyethylene glycol-polylactic acid (PEG- $b$-PLA) was performed by ring opening

5 polymerization of 3,4-Dimethyl-1,2-dioxane-2,5-dione (dilactide), using Tin(II) 2-ethylhexanoate

6 as catalyst (ratio catalyst/monomer: 1/2000) and the PEG-2000 as polymer chain initiator (ratio

$7 \quad$ PEG/lactic monomer 1/200)..$^{39} 40$

8 Briefly, 3,6-Dimethyl-1,4-dioxane-2,5-dione was recrystallized in toluene twice and dried under

9 vacuum for 2 days. Then, the 3,4-Dimethyl-1,2-dioxane-2,5-dione (10.8 g) were placed in a flask

10 and the PEG-2000 was added (1.0 g) along with the catalyst $(0.03 \mathrm{~g})$. The mixture was heated to

$11 \quad 150^{\circ} \mathrm{C}$ and stirred for $4 \mathrm{~h}$ under argon atmosphere. At the end of the reaction, the mixture was cooled

12 to ambient temperature. The crude reaction product was dissolved in dichloromethane and further

13 purified by precipitation in methanol twice. The obtained white polymer was dried under vacuum

14 for 2 days. 
1 The copolymer composition was quantified by dissolving a sample of the obtained diblock in

2 chloroform-d and analyzed by ${ }^{1} \mathrm{H}-\mathrm{NMR}$. The spectra were recorded with a $600 \mathrm{MHz}$ Varian Inova

3 Spectrometer (Palo Alto, California). Gel permeation chromatography (GPC) was performed on a

4 Breeze System from Waters (Waters, Milford, MA) equipped with a 2410 refractive index detector,

5 a 717 plus autosampler and a 1525 Binary HPLC pump and a Styragel HR3 column (5 HR3 column

$6 \quad(5 \mu \mathrm{m} 7.8 \mathrm{~mm} \times 300 \mathrm{~mm})$. Polystyrene standards were used for calibration. All samples were

7 filtered on a PTFE $0.2 \mu \mathrm{m}$ filters prior to injection. HPLC grade THF was used as eluent at a flow

8 rate of $1.0 \mathrm{~mL} / \mathrm{min}$.

$9 \quad$ Particles fabrication by nanoprecipitation.

10 Composition of the organic and aqueous phases

11 The same organic and aqueous phases were used for all the preparation methods. For the organic 12 phase, $60 \mathrm{mg}$ of PEG- $b$-PLA polymer were dissolved in $5 \mathrm{~mL}$ acetone, with the active compound at 13 different drug:polymer (D:P) ratio.

14 For the aqueous phase, we used water from a purification system (Milli-Q Q Gard-1, Millipore, $1518.2 \mathrm{M} \Omega . \mathrm{cm}, 25^{\circ} \mathrm{C}, \mathrm{TOC}<12 \mathrm{ppb}$ ). For all the formulations and all the fabrication processes, the 16 ratio between organic and aqueous phase was keep at 1 to 5 .

\section{Batch Nanoprecipitation (BN)}

$185 \mathrm{~mL}$ of organic phase were injected into $25 \mathrm{~mL}$ of MilliQ water under stirring in a $40 \mathrm{~mL}$ beaker 19 (450 rpm, Hotplate stirrer Mirak Thermolyne). The injection speed was set at $1.5 \mathrm{~mL} / \mathrm{min}$ with a 20 single syringe pump (Harvard apparatus, model 55-111) and needle gauge was 18G. The 21 nanosuspension was stored in Falcon ${ }^{\circledR}$ tubes at $4^{\circ} \mathrm{C}$ until further use.

\section{Confined Impinging Jet (Flash) Micromixer (CIJM)}

23 A CIJM was built according to reference ${ }^{41}$ and installed on the NanoAssemblr Benchtop 24 Instrument platform (Precision NanoSystems inc., Vancouver, BC, Canada) to inject and mix the 
1 organic and aqueous phases. Equal volumes of organic and aqueous phase $(5 \mathrm{~mL})$ were injected into

2 the micromixer, at a total speed of $9 \mathrm{~mL} / \mathrm{min}$ (output flow rate), unless otherwise indicated in the

3 text. The resulted nanosuspension was immediately quenched in a beaker with $20 \mathrm{~mL}$ of pure water

4 kept under constant magnetic stirring (for a final organic to aqueous phase volume ratio of 1:5). The

5 first and the last $100 \mu \mathrm{L}$ of the nanosuspension emerging from the mixing chamber were

6 systematically discarded before reaching the quenching solution. The obtained nanosuspension was

7 preserved in Falcon ${ }^{\circledR}$ tubes until further use.

$8 \quad$ Staggered Herringbone Micromixer Nanoprecipitation (SHMN)

9 The two phases $(1.67 \mathrm{~mL}$ of organic phase and $8.33 \mathrm{~mL}$ of aqueous phase) were injected into a 10 microfluidic cartridge (Precision NanoSystems inc., Vancouver, CB, Canada) at a total speed of 6 $11 \mathrm{~mL} / \mathrm{min}$, (unless otherwise indicated in the text) using the NanoAssemblr Benchtop Instrument

12 (Precision NanoSystems inc., Vancouver, CB, Canada).. The cartridge had been designed to

13 efficiently mix the two phases in a herringbone micromixer as previously described ${ }^{28}$. The process

14 was repeated tree times to obtain $30 \mathrm{~mL}$ of nanosuspension. Between each injection a washing step

15 was introduced to clean the microfluidic channels with acetone and water. The first and the last

$16100 \mu \mathrm{L}$ of the nanosuspension were systematically discarded. The obtained nanosuspension was

17 preserved in Falcon ${ }^{\circledR}$ tubes until further use.

\section{$18 \quad$ Nanoparticle characterizations}

$19 \quad$ Particle size and $\zeta$-potential

20 NPs suspensions (about $30 \mathrm{~mL}$ ) were dialysed in a dialysis tube with a MWCO of 50kDa (Spectrum

21 Laboratories, USA) during 6 hours, against $1 \mathrm{~L}$ of pure water. Particle size and size distribution of

22 dialyzed NPs were measured by dynamic light scattering (DLS) with a Malvern Zetasizer

23 instrument (Malvern Instruments, Worcestershire, UK) at $20^{\circ} \mathrm{C}$ and at a scattering angle of $173^{\circ}$.

24 Measurements were performed in triplicate. The dialyzed NP preparations were stored at $4^{\circ} \mathrm{C}$ until

25 further use. 
$\underline{\text { PEG content at the particle surface }}$

2 The total PEG content of NPs was determined by ${ }^{1} \mathrm{H}$ NMR. Briefly, $1.5 \mathrm{~mL}$ of the purified and

3 concentrated nanosuspension were lyophilised and resuspended in $0.75 \mathrm{~mL}$ of deuterated

4 chloroform. The total PEG content was determined by measuring the ratio of ethylene glycol proton

$5 \quad($ at $\delta=3.5) \mathrm{ppm})$ to the methane proton signal of lactic acid (at $\delta=5.16 \mathrm{ppm})^{13}$.

6 The determination of surface-grafted PEG content (\%w/w of Total PEG at the surface) and the PEG

7 surface density (PEG chain/nm $/ \mathrm{nm}^{2}$ ) was performed by ${ }^{1} \mathrm{H}$ NMR of the nanoparticles suspended in $\mathrm{D}_{2} \mathrm{O}$

8 using an internal standard ${ }^{13}$. Briefly, $20 \mathrm{~mL}$ of dialysed suspension were concentrated by tangential

9 flow filtration (TFF) using a hollow fiber filter module (PS, molecular weight cut-off (MWCO) 50

$10 \mathrm{kDa}$, surface area $28 \mathrm{~cm}^{2}$, Spectrum Laboratories, USA). The final volume of the suspension was

$115 \mathrm{~mL}$ after two purification/concentration cycles. Then, $0.5 \mathrm{~mL}$ of concentrated nanosuspension

12 were mixed with $0.5 \mathrm{~mL}$ of Deuterium oxide (with $1 \% \mathrm{w} / \mathrm{w}$ of 3-(trimethylsilyl)-1-propanesulfonic

13 acid as internal standard) and analyzed by ${ }^{1} \mathrm{H}$ NMR, in a Bruker AVII 400 spectrometer. The

14 calculation of the $\% \mathrm{w} / \mathrm{w}$ of total PEG at the surface of the nanoparticle and the PEG surface density

15 (PEG chain $\left./ \mathrm{nm}^{2}\right)$ were performed as described by Rabanel et al. ${ }^{13}$

16 Determination of Drug Loading (DL) and Encapsulation Efficiency (EE)

17 To evaluate the drug loading capacity of the NPs, $5 \mathrm{~mL}$ of the formulation was ultracentrifugated at

$184^{\circ} \mathrm{C}$ (Sorvall RC6, rotor SS-34, Thermo Scientific Canada) at $20000 \mathrm{rpm}$ (47 $800 \mathrm{rcf}$ ) for two

19 hours. The samples were centrifugated immediately after the preparation process. The conditions of

20 centrifugation were validated to ensure the total sedimentation of the NPs regardless of their

21 hydrodynamic diameter.

22 After centrifugation, $3 \mathrm{~mL}$ of the supernatant were taken out and frozen at $-20^{\circ} \mathrm{C}$ until the $\mathrm{HPLC}$ 23 analyses were performed (5 to 7 days after sample preparation). The pellet was reconstituted with 3

$24 \mathrm{~mL}$ of pure water and transferred into three Eppendorf tubes of $1.5 \mathrm{~mL}$ (previously weighed) for 25 lyophilisation. After lyophilisation, the pellets were weighed to determine the total NPs weight, to 
1 be used in the calculation of DL. The amount of drug present in the NPs was determined as the

2 difference between the total amount of drug used to prepare the NPs and the amount of drug present

3 in the supernatant.

4 The supernatant was diluted in mobile phase and injected into a Shimadzu Prominence (Shimadzu

5 USA Manufacturing Inc.) high performance liquid chromatography (HPLC) system composed of a

6 pump (LC-20A HT), and UV-VIS detector (SPD-20A), a column oven CTO-20A), a syringe

7 loading sample injector (SIL-20A) and a Hypersil GOLD PFP column (150 x $4.6 \mathrm{~mm}$ i.d., $5 \mu \mathrm{m}$ of

8 particle size). For the HPLC analysis of Theophylline, the mobile phase was MilliQ Water -

9 Methanol - Glacial Acetic Acid $(64: 35: 1 \mathrm{v} / \mathrm{v} / \mathrm{v})$ at a flow rate of $0.8 \mathrm{~mL} / \mathrm{min}, 20 \mu \mathrm{L}$ of injection

10 volume and column oven temperature was set at $30^{\circ} \mathrm{C}$. For Ketoprofen, the mobile phase was

11 Acetonitrile - MilliQ Water - Glacial Acetic Acid (90:110:1 v/v/v) at a flow rate of $1.0 \mathrm{~mL} / \mathrm{min}$ and

$1220 \mu \mathrm{L}$ of injection volume. The calibration curves for both Theophylline and Ketoprofen were found

13 linear between 2 and $20 \mu \mathrm{g} / \mathrm{mL}\left(\mathrm{r}^{2}=0.998\right.$ and 0.999 respectively).

14 To directly quantify the amount of drug encapsulated, the lyophilised NP pellet were dissolved with

$151 \mathrm{~mL}$ of Acetonitrile and further diluted in the same solvent to reach a concentration within the

16 linearity interval of the quantification method. This preparation was analyzed with a Varian Cary

17100 Bio UV-Visible Spectrophotometer (Varian Inc. city country). The wavelengths used for

18 detection were the following: $268 \mathrm{~nm}$ for Theophylline, $258 \mathrm{~nm}$ for Ketoprofen and $265 \mathrm{~nm}$ for

19 Cholecalciferol. The calibration curves for the three actives molecules were linear between 2 and 20

$20 \mu \mathrm{g} / \mathrm{mL}$.

21 The direct quantification of the drugs in the pellets (encapsulated drug) produced similar results

22 compared to the indirect quantification by determination of the non-encapsulated fraction in the

23 supernatant. This last approach is easier, faster and do not necessitate the extraction of the 
1 encapsulated drug from the polymer matrix and was therefore used in this study. Quantification in

2 the supernatant was used as a redundant quality check.

3 The encapsulation efficiency, EE, and the drug loading, DL, were calculated according to Equation

$4 \quad(1)$ and (2):

$5 E E(\%)=\frac{(\text { Mass of drug entrapped in the NP }) \times 100}{\text { Total mass of drug }}$

$6 \quad D L(\%)=\frac{(\text { Total mass of drug }- \text { mass of drug in supernatant }) \times 100}{\text { Total mass of NP }}$

7

$8 \quad$ Results and discussion

9 The diblock polymer was synthesized by ROP using Methoxy-PEG 2kDa as the chain initiator. This 10 synthesis route is much more efficient (yielding a $>90 \%$ of chains with a PEG segment attached 11 according to NMR results) than the alternative strategy relying on a coupling reaction between the 12 PEG chain and the polyester chains ${ }^{42}$. The ${ }^{1} \mathrm{H}$ NMR spectrum of the polymer after purification is 13 shown in Supplementary Information, Figure S1. NMR and GPC data allowed the calculation of the 14 number averaged and weight averaged molecular weight of the polymer, $M n$ and $M w$ respectively, 15 as well as polymer polydispersity. The resulting structural properties are summarized in Table 2.

16 Table 2. Structural properties of the polymer used in the present study

\begin{tabular}{|c|c|c|c|c|c|}
\hline \multirow[t]{2}{*}{ Polymer } & $\mathrm{M}_{\mathrm{n}}^{\mathrm{a}}$ & $\mathrm{M}_{\mathrm{w}}{ }^{\mathrm{a}}$ & $\mathrm{M}_{\mathrm{w}} / \mathrm{M}_{\mathrm{n}}{ }^{\mathrm{a}}$ & $\begin{array}{c}\text { Content of } \\
\text { PEG }^{\mathrm{c}}\end{array}$ & $\begin{array}{c}\mathrm{M}_{\mathrm{n}} \\
\left({ }^{1} \mathrm{H} \mathrm{NMR}\right)^{\mathrm{b}}\end{array}$ \\
\hline & {$[\mathrm{g} / \mathrm{mol}]$} & {$[\mathrm{g} / \mathrm{mol}]$} & & $\% \mathrm{w} / \mathrm{w}$ & {$[\mathrm{g} / \mathrm{mol}]$} \\
\hline PEG- $b$-PLA & 24200 & 34200 & 1.41 & 8.3 & 24000 \\
\hline
\end{tabular}

$17{ }^{a}$ Determined by GPC analysis. $M w / M n=$ Polydispersity index of the polymer.

$18{ }^{b}$ Calculated from peak intensity ratios of PEG (3.6 ppm) and PLA (5.2 ppm) by ${ }^{1} \mathrm{H} N M R$

$19{ }^{c}$ Calculated from NMR 
2 We first characterized the effect of the Drug : Polymer ratio (D:P) on the particle size for each

3 mixing method. The D:P was increased by keeping constant the concentration of the polymer in the

4 organic phase and increasing the drug concentration. Figure $2 \mathrm{~A}$ shows that the hydrodynamic

5 diameter $D_{\mathrm{H}}$ of the particles measured by DLS increases with D:P only for the very hydrophobic

6 drugs $(\log \mathrm{P}>7$, Cholecalciferol) and tend to be independent of D:P for more water soluble drugs

$7 \quad(\log \mathrm{P}<7)$. As shown in Fig. 2B, the hydrophobicity of the drug seems to control the final particle

8 size for the CIJM and SHM methods but has little to no impact in the BN process, a trend observed

9 at two different D:P ratios (10 and 20\%). Further insights into these observations will be provided

10 when analysing the effect of hydrophobicity on the drug loading and encapsulation efficiency. Of

11 importance, the size polydispersity index (PdI) did not significantly vary with D:P (Figure S2).

12 However differences were observed between fabrication processes, SHMN displaying slightly

13 higher size PdI compared to BN and CIJM.
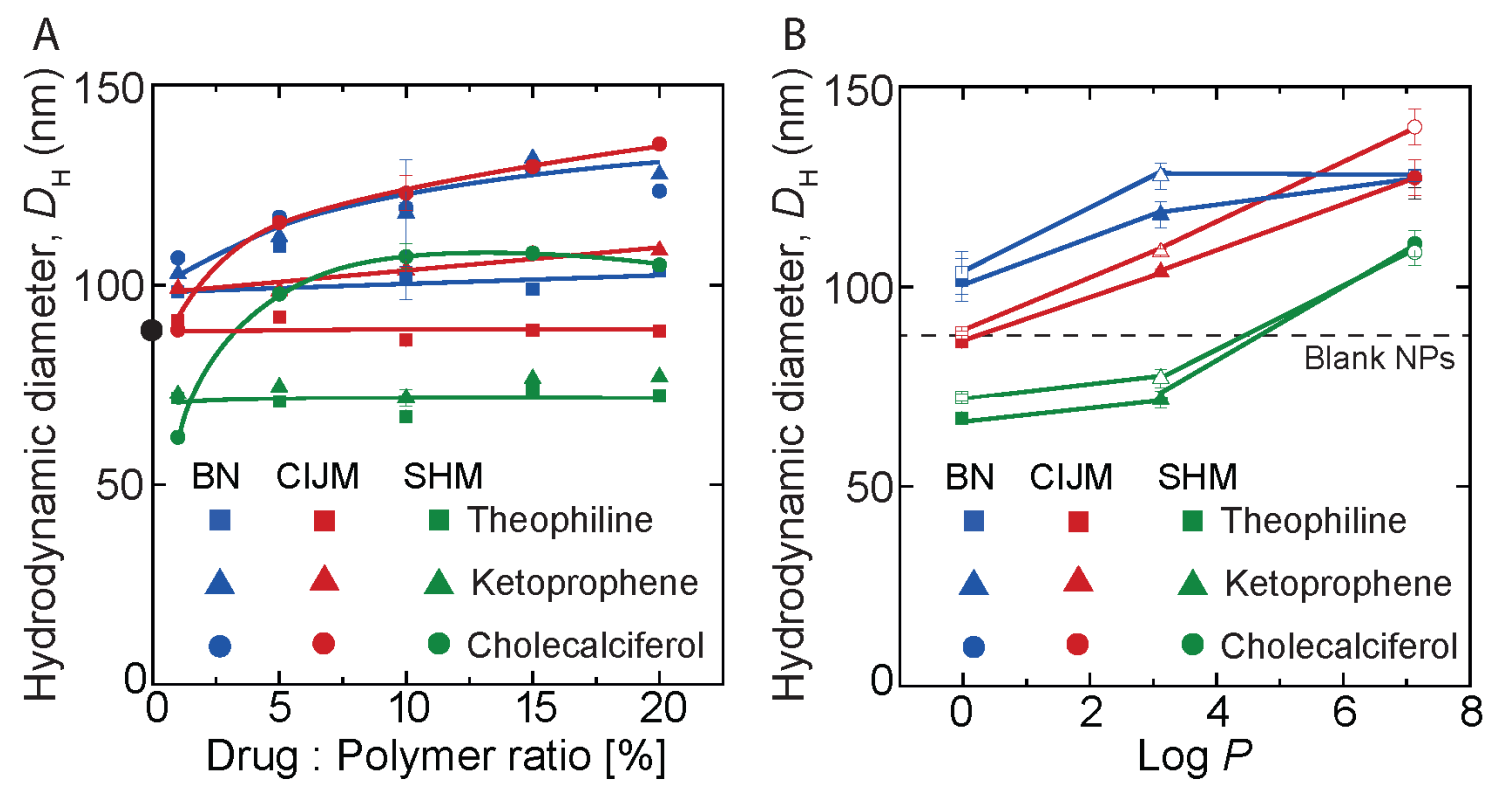

Figure 2. Hydrodynamic diameter, $D_{\mathrm{H}}$, of NPs prepared by different methods (A) Particle size according to the drug polymer ratio. Results are represented as means \pm S.D. of three independent experiments for D:P $=10 \% \mathrm{w} / \mathrm{w}$ only (B) Particle size $v s \log P$ of the encapsulated drug, solid symbols represent the formulation with $\mathrm{D}: \mathrm{P}=10 \% \mathrm{w} / \mathrm{w}$, while empty symbols correspond to $\mathrm{D}: \mathrm{P}=$ $20 \% \mathrm{w} / \mathrm{w}$. 
2 An additional trend can be extracted from this first series of experiments. It appears that

3 independently of the D:P ratio or $\log \mathrm{P}, D_{\mathrm{H}}$ tends to decrease from one fabrication method to the

4 other in the following order: $\mathrm{BN}>\mathrm{CIJM}>$ SHM. Explaining this trend requires a deep analysis of

5 the physics of the mixing process and a estimation of the different time scales involved in the NP

6 formation. During the nanoprecipitation process, particles are formed by diffusion-limited

7 coalescence ${ }^{43-44}$ of smaller particles until stability is reached. Stability can be provided by

8 adsorption of surfactant molecules present in the suspension or by the polymer forming the particle

9 itself (if it is a diblock polymer for example). The characteristic time associated to the coalescence

10 process, $\tau_{\mathrm{cls}}$, is defined as $\tau_{\mathrm{cls}}=3 / 8(\eta / k T)\left(M_{w} / N_{A} C_{p}\right)$ where $C_{\mathrm{p}}$ is the polymer mass

11 concentration, $M_{\mathrm{w}}$ its molar mass, $\eta$ the medium viscosity and $N_{\mathrm{A}}$ the Avogadro number. In order

12 for the polymer chains to coalesce, the organic and aqueous phases need to be quickly mixed in

13 order to reach supersaturation (of the polymer and the drug). The mixing time, $\tau_{\text {mix }}$ which defines

14 the time scale for complete mixing should be smaller than the residence time, $\tau_{\text {res}}$, of the mixture in

15 the mixing chamber. The residence time can be estimated (upper value) by $\tau_{\text {res }}=V_{m} / Q_{t}$ where $V_{\mathrm{m}}$

16 and $Q_{\mathrm{t}}$ are the mixing volume and the total flow rate, respectively. In Table 3, we provide estimates

17 of each of the characteristic times for the operating conditions used in the present study. For batch nanoprecipitation (BN), we estimated the upper boundary of the mixing time experimentally using a

$19 \mathrm{pH}$-titration method. This estimate is expected to be significantly higher to the real mixing time

20 since the probe size ( $\mathrm{pH}$ electrode) and the injection nozzle used for the nanoprecipitation could not

21 be located exactly at the same position in the beaker and also because the response time of the $\mathrm{pH}-$

22 meter is of the order of 1 second. For the CIJM, Johnson and Prud'homme have provided a detailed

23 calculation of $\tau_{\text {mix }}$ in the low Reynolds regime (which is our case since Re $=100-3000$ ). ${ }^{45}$ For the

24 SHM, we used the calculations provided by Kee et al. for the same geometry. ${ }^{30}$ 
2 Table 3. Estimates of the characteristic times involved in the different mixing methods used

\begin{tabular}{cccc}
\hline $\begin{array}{c}\text { Fabrication } \\
\text { Method }\end{array}$ & $\begin{array}{c}\text { Residence time, } \tau_{\text {res }} \\
{[\mathrm{ms}]}\end{array}$ & $\begin{array}{c}\text { Coalescence time, } \tau_{\text {cls }} \\
{[\mathrm{ms}]}\end{array}$ & $\begin{array}{c}\text { Mixing time, } \tau_{\text {mix }} \\
{[\mathrm{ms}]}\end{array}$ \\
\hline BN & $1.10^{6}$ & 80 & $1.10^{3}-10.10^{3}$ \\
CIJM & $100-1000$ & 80 & $40-900$ \\
SHMN & $20-200$ & 80 & $10-100$ \\
\hline
\end{tabular}

3

4 We can see from Table 3 that the used operating conditions were favorable to achieve complete 5 mixing $\left(\tau_{\text {res }}>\tau_{\text {mix }}\right)$. Under these operating conditions, the final particle size, which in our case is 6 close to the hydrodynamic size since the thickness of the PEG corona is much smaller than the 7 particle core, can be described by the following equation: ${ }^{43}$

$$
R_{p}=R_{p o}(1+k)\left(1+\frac{\tau_{m i x}}{\tau_{c l s}}\right)^{1 / 3}
$$

9 where $R_{\mathrm{p}}$ is the final radius of the particle, $R_{\mathrm{po}}=\left(3 M_{\mathrm{p}} /\left(4 \pi N_{\mathrm{A}} \rho\right)\right)^{1 / 3}$ and $k$ is the ratio of the total 10 initial surface area of the polymer particles and the maximum area surfactant molecules can cover.

11 Within this framework, we performed a new series of experiments which we compiled with 12 experimental data of Figure 2A. In this series, particle size was monitored at different injection flow 13 rates and rescaled as presented in Figure $3 \mathrm{~A}$. Due to the uncertainties in determining $\tau_{\text {mix }}$ for the BN 14 method, we used Eq. 3 to calculate an average value of $\tau_{\operatorname{mix}} / \tau_{\text {cls }}$ for the three tested drugs. 

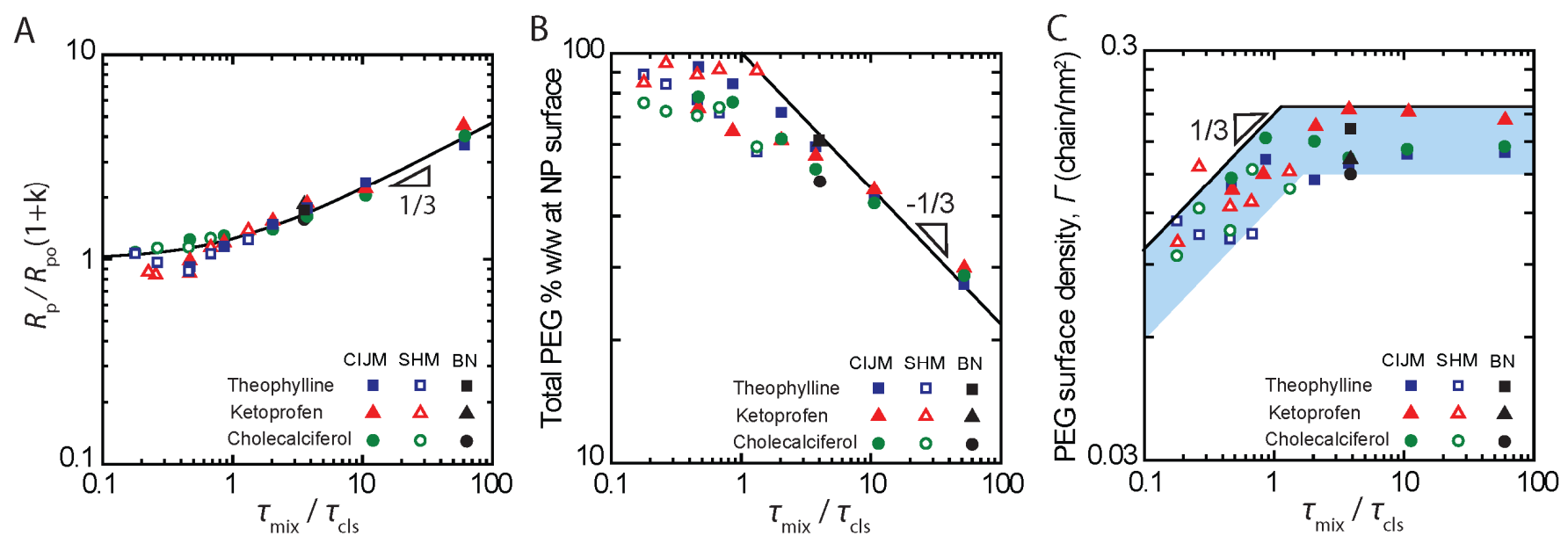

Figure 3: A) Evolution of the particle size with the rescaled mixing time shows that the diffusion-limited coalescence model (Eq. 3) describes adequately the experimental data. B) Relative distribution of PEG chains between the core and the surface of the particle. In the slow mixing regime $\left(\tau_{\text {mix }} / \tau_{\mathrm{cls}}>1\right)$, PEG chains tend to accumulate in the core of the particles, in agreement with a pure coalescence process with no chain rearrangement while in the fast mixing regime, rapid rearrangement of PEG chains allows to maintain PEG content at the surface constant. C) The calculated PEG chain surface density appears to be constant in the slow mixing regime, in agreement with a diffusion-limited coalescence process with slow PEG chain rearrangement (see text for details). All data were collected at $\mathrm{D}: \mathrm{P}=10 \% \mathrm{w} / \mathrm{w}$.

1

2 Figure $3 \mathrm{~A}$ shows that the experimental data collected for all the formulations tested can be

3 collapsed into one master curve obtained from Eq. 3. At high $\tau_{\text {mix }} / \tau_{\mathrm{cls}}$ ratio, the final particle radius

4 follows the simple power law $R_{\mathrm{p}} \sim\left(\tau_{\text {mix }} / \tau_{\mathrm{cls}}\right)^{1 / 3}$ which is characteristic of the coalescence process. At

5 low $\tau_{\text {mix }} / \tau_{\text {cls }}$, mixing of the organic and aqueous phases is extremely fast and therefore the particle

6 size tends towards the limit size $R_{\mathrm{po}}$. As we will immediately show, this universal behavior extends

7 to other properties such as PEG distribution in the particles.

8 To demonstrate this, we measured the relative distribution of PEG between the shell and the core of

9 the NP using NMR spectroscopy. ${ }^{13}$ In Figure 3B we report the fraction of PEG (expressed as a

$10 \% \mathrm{w} / \mathrm{w}$ ) present at the NP surface and in Figure 3C the corresponding PEG surface density

11 calculated under the assumption of a particle core diameter close to the hydrodynamic diameter (a

12 valid assumption for particle radii much larger than the PEG chains characteristic size). We can see

13 from these two figures that in the slow mixing regime $\left(\tau_{\text {mix }} / \tau_{\mathrm{cls}}>1\right)$, PEG $\% \mathrm{w} / \mathrm{w}$ decreases 
1 significantly while the PEG surface density, $\Gamma$, remains fairly constant, independently of the drug.

2 On the other side, in the fast mixing regime $\left(\tau_{\text {mix }} / \tau_{\text {cls }}<1\right)$, surface PEG \%w/w appears to decrease

3 with a weak power law while PEG surface coverage remains constant. These differences in

4 behavior between PEG distribution and PEG surface density are reminiscent of a coalescence

5 process with or without rearrangement of the PEG blocks.

6 When polymer blocks are free to rearrange during particles coalescence, PEG blocks remain 7 entirely at the formed particle surface and therefore PEG $\% \mathrm{w} / \mathrm{w}$ is expected to remain constant and 8 equal to $100 \% \mathrm{w} / \mathrm{w}$. The PEG surface density $\Gamma$, on the other hand, should increase slightly simply 9 because a particle resulting from a coalescence event has a smaller surface area than the sum of the 10 areas of the initial two colliding particles. To explain further this result, let's consider first the 11 coalescence of two identical particles of size $R_{1}$ and PEG surface density $\Gamma_{1}$ which we call $\mathrm{P}_{1}\left[R_{1}\right.$, $\left.12 \Gamma_{1}\right]$. After coalescence, the resulting particle $\mathrm{P}_{2}\left[R_{2}, \Gamma_{2}\right]$ has a diameter of $R_{2}=2^{1 / 3} R_{1}$ and $\Gamma_{2}=2^{1 / 3} \Gamma_{1}$ 13 if all PEG chains can rearrange and remain at the particle surface. The coalescence process 14 continues such that $\mathrm{P}_{3}=\mathrm{P}_{2}+\mathrm{P}_{1}, \ldots, \mathrm{P}_{\mathrm{n}}=\mathrm{P}_{\mathrm{n}-1}+\mathrm{P}_{1}$. Simple calculations show that $R_{\mathrm{n}}=\mathrm{n}^{1 / 3} R_{1}$ and $\Gamma_{\mathrm{n}}$ $15=\mathrm{n}^{1 / 3} \Gamma_{1}$ which can be expressed in terms of measurable quantities remembering that $\mathrm{n}=\tau_{\text {mix }} / \tau_{\mathrm{cls}}$.

16 Therefore, in the case of coalescence with chain rearrangement, $\Gamma$ is expected to follow the power 17 law $\Gamma \sim\left(\tau_{\text {mix }} / \tau_{\mathrm{cls}}\right)^{1 / 3}$. One can easily verify that in this regime, PEG \%w/w at the NP surface is 18 indeed equal to $100 \% \mathrm{w} / \mathrm{w}$ remembering that $\mathrm{PEG} \%\left(\mathrm{P}_{\mathrm{n}}\right)=\Gamma_{\mathrm{n}} R_{\mathrm{n}}{ }^{2} /\left(\Gamma_{\mathrm{n}-1} R_{\mathrm{n}-1}{ }^{2}+\Gamma_{1} R_{1}{ }^{2}\right)$. We can see in 19 Figure $3 \mathrm{~B}$ and $\mathrm{C}$ that $\mathrm{PEG} \% \mathrm{w} / \mathrm{w}$ at the NP surface is constant and close to $100 \%$ in the fast mixing 20 regime $\left(\tau_{\text {mix }} / \tau_{\text {cls }}<1\right)$ where the NP size is the smallest, suggesting that fast chain rearrangement is 21 occurring, which is also confirmed by the surface density data which demonstrated a weak power 22 law increase with an exponent of $1 / 3$.

23 During the coalescence process with no or slow rearrangement of polymer chains, PEG blocks are trapped in between the particles when they first encounter and remain inside the resulting particle 
1 core (see Figure 4). Following the same line on thoughts, we can see that when two particles $\mathrm{P}_{1}\left[R_{1}\right.$,

$2 \Gamma_{1}$ ] encounter, the surface area, $\mathrm{S}_{2}$ of $\mathrm{P}_{2}$ is $\mathrm{S}_{2}=2{ }^{2 / 3} \mathrm{~S}_{1}$. The amount of trapped PEG blocks is

3 therefore $2 \Gamma_{1} R_{1}{ }^{2}-2^{1 / 3} \Gamma_{1} R_{1}^{2}$ which leads to the final expression $\Gamma_{2}=\Gamma_{1}$. The associated PEG \%w/w

4 at $\mathrm{P}_{2}$ surface is $\mathrm{PEG} \%\left(\mathrm{P}_{2}\right)=\Gamma_{1} R_{2}^{2} /\left(2 \Gamma_{1} R_{1}{ }^{2}\right)=2^{-1 / 3}$. For a particle $\mathrm{P}_{\mathrm{n}}$, one can immediately see that $\Gamma_{\mathrm{n}}$

$5=\Gamma_{1}$ and PEG\% $\left(\mathrm{P}_{\mathrm{n}}\right)=\mathrm{n}^{-1 / 3}=\left(\tau_{\text {mix }} / \tau_{\mathrm{cls}}\right)^{-1 / 3}$. As can be seen in Figure $3 \mathrm{~B}$ and $\mathrm{C}$, these predictions are

6 in agreement with our experimental data in the slow mixing regime $\left(\tau_{\text {mix }} / \tau_{\mathrm{cls}}>1\right)$, where large

7 particles are formed. These results are also consistent with the fact that in large particles of radius $R$,

8 polymer chains rearrangement is indeed slower since the characteristic time for a chain to diffuse

9 from the core to the surface of the particle is $\sim R^{2} / D, D$ being the reptation diffusion coefficient in

10 the melt.

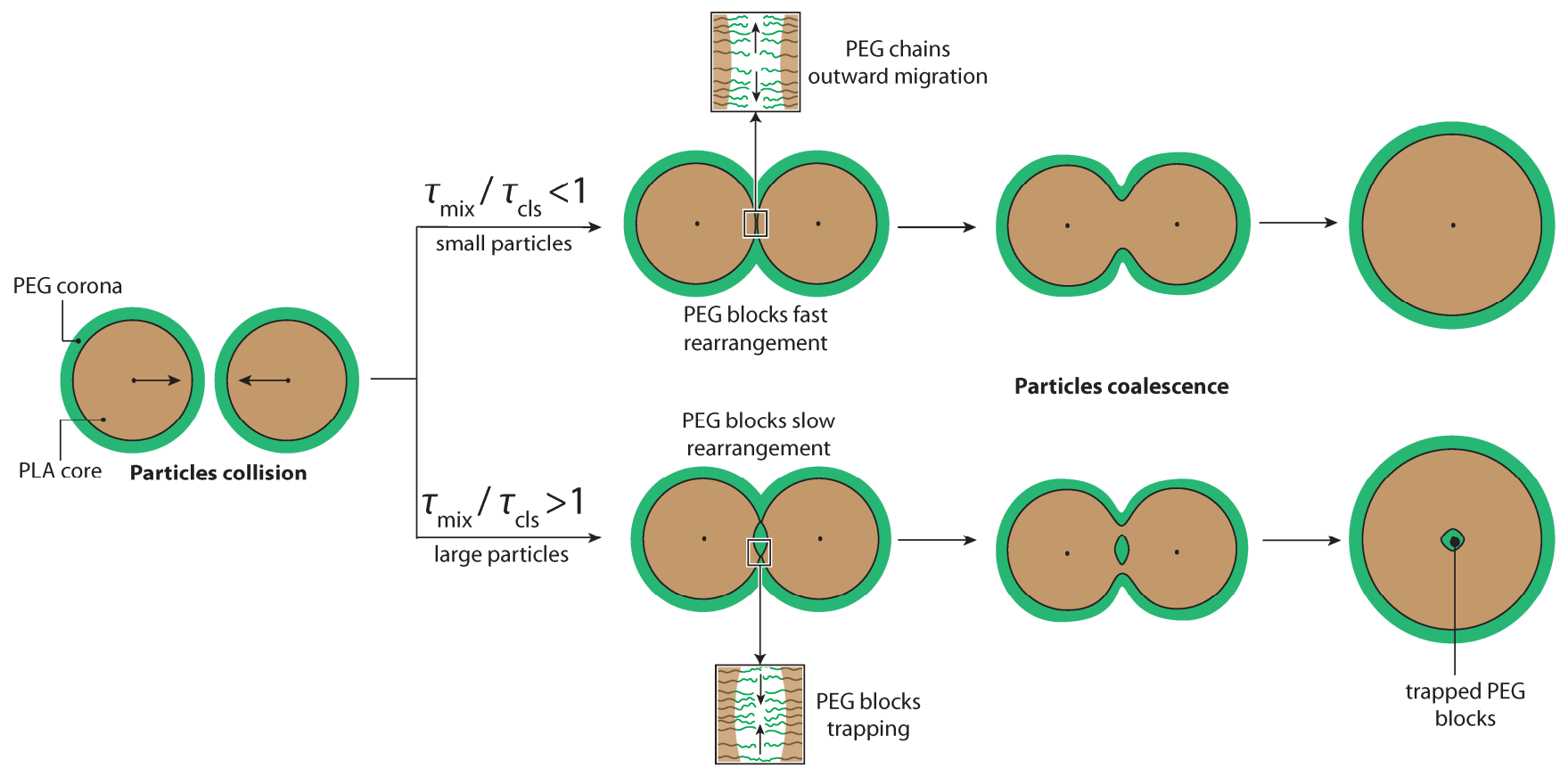

Figure 4: Schematic representation of the different scenarios occurring during the coalescence of two PLA-bPEG particles. Particles contact can either involve PEG blocks fast rearrangement in the contact area in order to facilitate the fusion between the two hydrophobic PLA cores or involve slow rearrangement of PEG blocks leading to their entrapment inside the core of the particles. The fast rearrangement scenario is predominant in the fast mixing regime during which small particles are formed while the slow rearrangement scenario is characteristic of the slow mixing regime.

11 
1 This analysis of the nanoparticle structure and its scaling with mixing conditions ignores the

2 presence of the drug in the formulation. As shown in Fig. S3 and Table S2, PEG distribution and

3 PEG surface density do not depend significantly on D:P for all the drugs tested. This is a strong

4 difference with other types of self-assembled nanostructures such as filomicelles, where dramatic

5 structural changes have been reported during encapsulation. ${ }^{46}$

6 The particles formed via the process described before were found to be stable over time with a

7 particle size change of less than $10 \%$ over 7 days and zeta potential variation of less than $15 \%$ over

87 days of monitoring (see Supplementary Information Table S3 to S5).

9 Since the prepared nanosuspensions are meant to be used as drug delivery systems, we evaluated

10 their capacity to encapsulate different drugs. Many researchers ${ }^{4,47-49}$ have demonstrated that PLA- $b$ -

11 PEG polymers are well suited for the encapsulation of a wide range of active compounds but to our

12 knowledge, studies rationalizing the relationship between their loading capacity and the fabrication

13 method is still lacking. In Figure 5A we show the variation of the drug loading, DL, as a function of

$14 \mathrm{D}: \mathrm{P}$ at fixed mixing conditions $\left(\tau_{\mathrm{mix}} / \tau_{\mathrm{cls}}=2-4\right)$. As shown in Figure 5A, CIJM produces

15 significantly higher $\mathrm{EE}$ and DL compared to the other two mixing techniques for

16 Theophylline. This difference disappears for the more hydrophobic drugs Ketoprofen and

17 Cholecalciferol. For these two last drugs and all three fabrication methods, the relationship

18 between DL and D:P is linear indicating that saturation of the drug inside the NP has not been

19 reached within the window of D:P covered. 

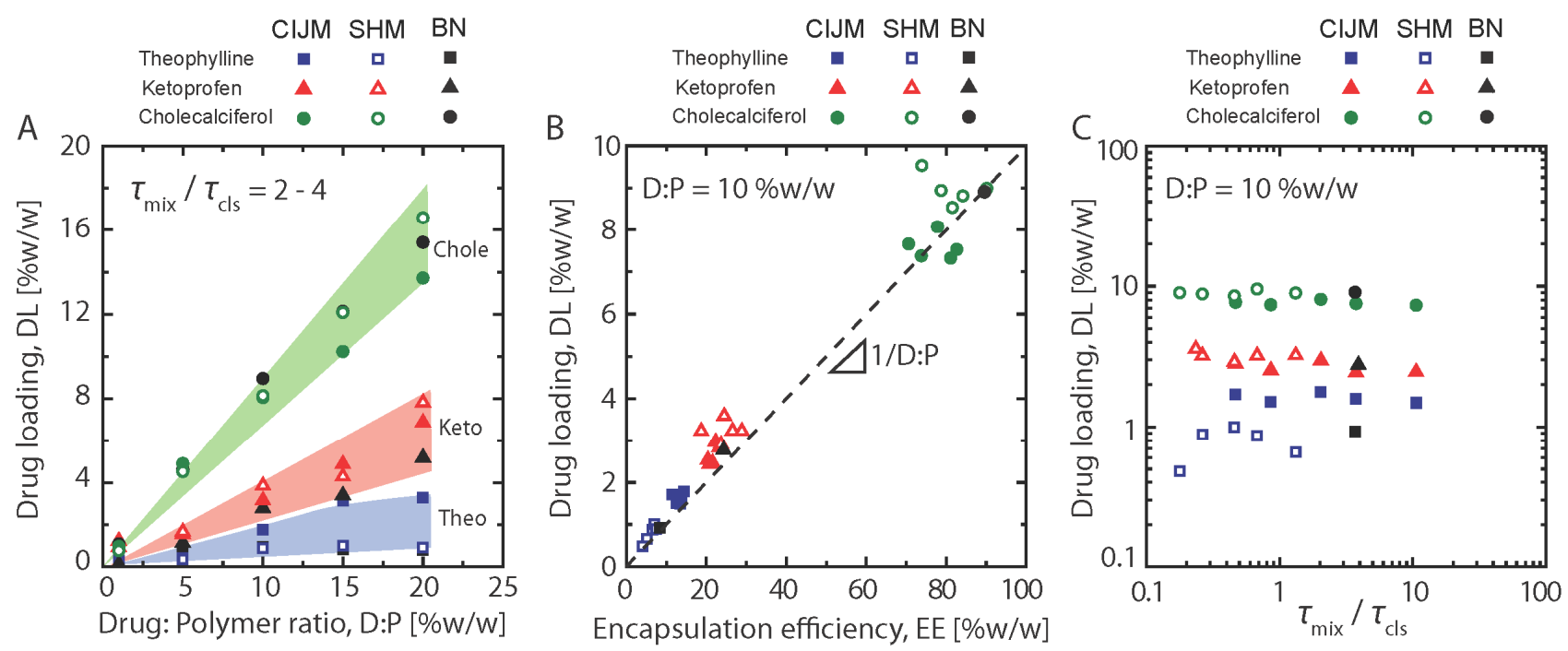

Figure 5: Encapsulation of drugs in PLA-b-PEG nanoparticles. (A) Drug loading shows a consistent linear increase with $D: P$ for the most hydrophobic drugs while reaching a saturation plateau for the most hydrophilic drug (theophylline). (B) Drug loading is slightly affected by the fabrication method but still increases linearly with EE as expected. (C) DL shows a very weak dependence on mixing time which is reminiscent of a coagulation process. In the case of theophylline, the CIJM method leads to overloaded particles (see text for details).

1 On the contrary, theophylline shows a saturation plateau reached at D:P $=10-15 \% \mathrm{w} / \mathrm{w}$. The DL

2 value of this plateau is $0.8 \% \mathrm{w} / \mathrm{w}$ for $\mathrm{BN}$ and $\mathrm{SHM}$ and $1.7 \% \mathrm{w} / \mathrm{w}$ for CIJM. As expected, the more

3 hydrophobic the drug is, the higher is its DL, a trend extensively reported for nanosuspensions

4 produced by BN. ${ }^{50}$ Our results demonstrate that this trend is still preserved with other fabrication

5 methods although with some subtle differences that we will explain. In Figure 5B, we show the

6 evolution of DL vs EE at fixed D:P $=10 \% \mathrm{w} / \mathrm{w}$ but different mixing conditions. As expected, DL

7 increases linearly with EE and as before, increases with the hydrophobicity $(\log \mathrm{P})$ of the drug. A

8 distinctive result is the significant differences in DL from one fabrication method to another.

9 Indeed, for theophylline ( $\log \mathrm{P}=0$, hydrophilic), the DL and EE values are systematically smaller

10 for the SHM and BN compared to the CIJM. For ketoprofen, the differences in DL are much

11 smaller and all three methods produced NPs with a DL between 2.5-3.5\%w/w and EE \%w/w

12 between $18.5-29.5 \% \mathrm{w} / \mathrm{w}$. For the most hydrophobic drug, cholecalciferol $(\log \mathrm{P}=7.13)$, NP

13 generated with the CIJM appear to have a slightly lower DL (DL $=7.2-8 \% \mathrm{w} / \mathrm{w}$ ) compared to the

$14 \mathrm{BN}$ and $\mathrm{SHM}$ methods $(\mathrm{DL}=8.5-9.5 \% \mathrm{w} / \mathrm{w})$. For ketoprofen and cholecalciferol, these small 
1 differences are partly due to the weak dependence of DL with mixing time (see Figure 5C). Given

2 that theophylline maximum loading is reached at D:P $=10 \% \mathrm{w} / \mathrm{w}(\mathrm{DL}=0.8 \% \mathrm{w} / \mathrm{w}$ for SHM and

$3 \mathrm{BN}$ and $\mathrm{DL}=1.7 \% \mathrm{w} / \mathrm{w}$ for CIJM), it is important to identify which of these values is closer to the

4 thermodynamic value.

5 As suggested by Kumar and Prud'Homme ${ }^{51}$, the maximum drug loading of polymeric NPs can be

6 calculated using the following expression of the variation of the mixing free energy $\Delta G$ of the drug

7 and polymeric NP core (PLA):

8

$$
\Delta G=\Re T\left(\operatorname{Ln}\left(\phi_{D r u g}\right)+\left(1-\frac{V_{m}^{\text {Drug }}}{V_{m}^{P L A}}\right) \phi_{P L A}+\chi \phi_{P L A}^{2}\right)+\frac{2 \gamma_{e f f}}{R} \quad \text { Eq. } 4
$$

9 where $\Phi$ is the volume fraction of the drug or PLA, $V_{m}$ the molar volume, $\chi$ the Flory-Huggins 10 (FH) interaction parameter, $\gamma_{\text {eff }}$ the interfacial energy of the particle and $R$ the particle core radius.

11 The first term in brackets represents the mixing energy directly derived from FH theory while the 12 second term is the Laplace interfacial contribution. The equilibrium condition, $\Delta G=0$ allows to 13 calculate $\Phi_{\text {Drug }}$ and DL remembering that $\mathrm{DL}^{-1} \approx 1+\left(\Phi_{\mathrm{PLA}} / \Phi_{\text {Drug }}\right) .\left(\rho_{\mathrm{PLA}} / \rho_{\text {Drug }}\right) .(1-\alpha)^{-1}, \alpha$ being the 14 total PEG content in the polymer $(8.3 \% \mathrm{w} / \mathrm{w})$. It is important to note that Eq. 4 is derived for a core 15 (PLA)- shell (PEG) particle which corresponds to the structure obtained at short mixing time. Using 16 the parameters provided in SI, we obtained a $\mathrm{DL}=0.76 \% \mathrm{w} / \mathrm{w}$ which is very close to the 17 experimental value $(\mathrm{DL}=0.8 \% \mathrm{w} / \mathrm{w})$ measured for the $\mathrm{BN}$ and $\mathrm{SHM}$ fabrication methods. 18 Therefore, for theophylline, the CIJM allows to reach a supersaturated state or overloading of the 19 NP. Since no evidence of drug crystallization in the NPs could be found by electron microscopy or 20 differential calorimetry (not shown), the only plausible explanation for the drug overloading is the 21 presence of PEG domains inside the NP core able to retain higher content of drug molecules 22 compared to PLA. Assuming a PEG domain size ten times smaller than the NP size, we can 23 estimate the maximum drug loading in the PEG domain using Eq. 4 using PEG instead of PLA. The 24 result (assuming that no water molecules are trapped in the PEG domain) gives a $\mathrm{DL}=1.5 \% \mathrm{w} / \mathrm{w}$, 
1 twice the value obtained for PLA, which confirms that, even if PEG domain are small in the particle

2 core (so their interfacial tension is very high, which should limit the encapsulation of small

3 molecules), they can still concentrate drug molecules and increase the total DL of the particle.

4 Besides the slight differences produced by the different fabrication methods, force is to recognize

5 that DL varies very weakly with the mixing time (Figure 5C). If we consider the experimental data

6 obtained with the CIJM method, we see that $\mathrm{DL} \approx\left(\tau_{\mathrm{mix}} / \tau_{\mathrm{cls}}\right)^{\beta}$ with $\beta=0.05-0.07$ for all three drugs.

7 Such behavior is a direct consequence from the coagulation process since the final particles are

8 formed through the coagulation of smaller particles, all having the same DL.

9 Data presented in Figure 5 support the main assumption that DL and EE are mostly determined by 10 the relative affinity of the drug to water and the polymer, i.e. by its partition coefficient $\mathrm{K}_{\mathrm{p}}=$ $11[\text { Drug }]_{\mathrm{p}} /[\text { Drug }]_{\mathrm{w}}$. Simple calculations show that, below particle saturation, DL varies linearly with 12 D:P as DL $\approx \mathrm{K}_{\mathrm{p}} \Phi .(\mathrm{D}: \mathrm{P})$ when $\mathrm{K}_{\mathrm{p}} \Phi<<1$, ( $\Phi$ being the volume fraction of particles) while $\mathrm{DL} \approx \mathrm{D}: \mathrm{P}$ 13 when $\mathrm{K}_{\mathrm{p}} \Phi>>1$, which is consistent with our observations.

14 If the present analysis suggests an interesting manner to determine $\mathrm{K}_{\mathrm{p}}$ for many drug/polymer pairs, 15 it also suggests that other parameters similar to $\mathrm{K}_{\mathrm{p}}$, such as the water/octanol partition coefficient 16 (LogP) could be used to predict DL. In Figure 6 we represented the evolution of EE and DL with $17 \quad \log \mathrm{P}$ at constant D:P. The results exhibit a quasi linear increase for both EE and DL. This 18 observation may help to qualitatively predict the encapsulation efficiency of different molecules 19 using the partition coefficient as sole predictor. 

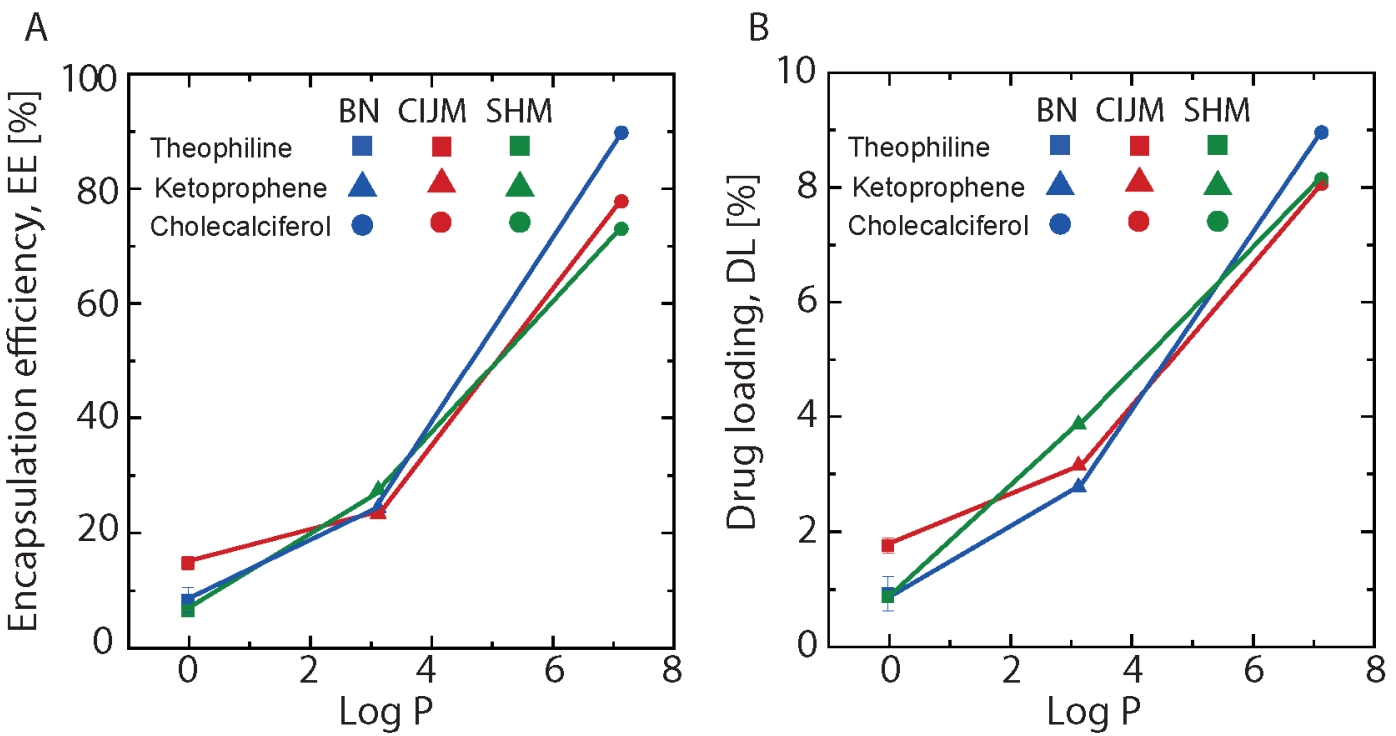

Figure 6. The encapsulation of an active compound is primarily determined by its partition coefficient. Both the encapsulation efficiency EE (A) and the drug loading DL (B) increase with the $\log \mathrm{P}$ of the drug independently of the fabrication method used. $\mathrm{D}: \mathrm{P}=10 \%$.

1

2 To summarize the present findings, we have shown that the structure of core/shell particles formed

3 via diffusion/coagulation is strongly dependent of the mixing conditions. On the other hand, the

4 drug loading and encapsulation efficiency depend solely on the relative affinity of the drug to the

5 polymer. Our results present marked differences to studies using polymeric micelles made of diblock polymer of much smaller molecular weight. In the case of polymeric micelles, fast rearrangement of polymer chains occurs at each coalescence event allowing PEG blocks to protrude towards the aqueous medium exclusively, independently of the mixing conditions. As a consequence, PEG surface coverage in polymer micelles is expected to increase with mixing time and PEG is expected to be absent from the core of the micelle independently of the mixing conditions. As we have shown such behavior is still preserved with high molecular weight polymers only in the fast mixing regime, where small particles are produced.

\section{Conclusions}

We studied the impact of the fabrication method of polymeric NPs made of PLA-b-PEG polymer on their final structure and capacity to encapsulate active compounds. The characterizations accomplished in this study demonstrate that the fabrication method defines the mixing time scale 
range available for a defined set of operating conditions. As already reported for polymeric

2 micelles, the mixing time appeared to be the main predictor of the NP size, size polydispersity as

3 well as amount and distribution of surface PEG. A key difference between polymeric micelle and

4 the NPs produced in this study is the change in particle core composition with mixing conditions.

5 We found that as mixing time was longer, the particles core is larger and richer in PEG which can

6 help to improve the encapsulation of more hydrophilic compounds. Finally, we showed that the

7 encapsulation of drugs can be predicted by the Flory-Huggins theory of mixing considering the NPs

8 interfacial energy, as reported for polymeric micelles. Even though this theory is powerful, we

9 showed that the $\log \mathrm{P}$ of the encapsulated molecules, is a strong predictor of drug encapsulation

10 efficacy. Altogether, our data showed that for a given drug, drug loading is controlled by D:P while

11 the drug structure (PEG\% at the NP surface) is independently controlled via the mixing time.

12 The information provided in this study delineates rational guidelines for the selection of fabrication

13 methods to prepare polymeric NPs, taking into account not only the properties of the active

14 molecule encapsulated but also the characteristics the NP.

\section{Supporting information}

17 Polymer ${ }^{1} \mathrm{H}-\mathrm{NMR}$, NP fabrication protocol, size PdI measurements, PEG surface coverage, 18 parameters used in Eq. 4 and NP stability data (Size and Zeta potential).

\section{Authors information}

\section{Corresponding authors}

21 Tel. 514 343-2470; Fax. 514 343-2102; E-mail: xavier.banquy@umontreal.ca; jean-

22 michel.rabanel@iaf.inrs.ca

\section{$23 \quad$ Note}

24 The authors declare no competing financial interests 


\section{Acknowledgments}

T.R acknowledges the Consejo Nacional de Ciencia y Tecnología (CONACYT, México) and the Ministère de l'Éducation, de l'Enseignement Supérieur et de la Recherche (Quebec, Canada) for the financial supports. XB is grateful for the financial support from the CRC program and CIHR. AGA is grateful to the TransMedTec Institute for the HQP fellowship. TEM imaging was performed by the "Centre de Caractérisation Microscopie des Matériaux" of the École Polytechnique (Montréal, Québec, Canada) with the help of Jean-Philipe Massé. Alexander Cunningham is acknowledged for his help in GPC analysis.

\section{References}

1. Keck, C. M.; Müller, R. H., Drug nanocrystals of poorly soluble drugs produced by high pressure homogenisation. European Journal of Pharmaceutics and Biopharmaceutics 2006, 62 (1), 3-16.

2. Merisko-Liversidge, E. M.; Liversidge, G. G., Drug nanoparticles: formulating poorly watersoluble compounds. Toxicologic pathology 2008, 36 (1), 43-48.

3. Alexis, F.; Pridgen, E.; Molnar, L. K.; Farokhzad, O. C., Factors affecting the clearance and biodistribution of polymeric nanoparticles. Molecular pharmaceutics 2008, 5 (4), 505-515.

4. Soppimath, K. S.; Aminabhavi, T. M.; Kulkarni, A. R.; Rudzinski, W. E., Biodegradable polymeric nanoparticles as drug delivery devices. Journal of controlled release 2001, 70 (1), 1-20.

5. Rabanel, J.-M.; Faivre, J.; Tehrani, S. F.; Lalloz, A.; Hildgen, P.; Banquy, X., Effect of the Polymer Architecture on the Structural and Biophysical Properties of PEG-PLA Nanoparticles. ACS applied materials \& interfaces 2015, 7 (19), 10374-10385.

6. Elsabahy, M.; Wooley, K. L., Design of polymeric nanoparticles for biomedical delivery applications. Chemical Society Reviews 2012, 41 (7), 2545-2561.

$7 . \quad$ Bertrand, N.; Leroux, J.-C., The journey of a drug-carrier in the body: An anatomophysiological perspective. Journal of Controlled Release 2012, 161 (2), 152-163.

8. Singh, R.; Lillard, J. W., Nanoparticle-based targeted drug delivery. Experimental and molecular pathology 2009, 86 (3), 215-223.

9. Redhead, H.; Davis, S.; Illum, L., Drug delivery in poly (lactide-co-glycolide) nanoparticles surface modified with poloxamer 407 and poloxamine 908: in vitro characterisation and in vivo evaluation. Journal of Controlled Release 2001, 70 (3), 353-363.

10. Honary, S.; Zahir, F., Effect of zeta potential on the properties of nano-drug delivery systems-a review (Part 1). Tropical Journal of Pharmaceutical Research 2013, 12 (2), 255-264.

11. Xiao, K.; Li, Y.; Luo, J.; Lee, J. S.; Xiao, W.; Gonik, A. M.; Agarwal, R. G.; Lam, K. S., The effect of surface charge on in vivo biodistribution of PEG-oligocholic acid based micellar nanoparticles. Biomaterials 2011, 32 (13), 3435-3446.

12. Owens, D. E.; Peppas, N. A., Opsonization, biodistribution, and pharmacokinetics of polymeric nanoparticles. International Journal of Pharmaceutics 2006, 307 (1), 93-102.

13. Rabanel, J.-M.; Hildgen, P.; Banquy, X., Assessment of PEG on polymeric particles surface, a key step in drug carrier translation. Journal of Controlled Release 2014, 185, 71-87. 
14. Al-Hanbali, O.; Rutt, K. J.; Sarker, D. K.; Hunter, A. C.; Moghimi, S. M., Concentration dependent structural ordering of poloxamine 908 on polystyrene nanoparticles and their modulatory role on complement consumption. Journal of nanoscience and nanotechnology 2006, 6 (9-1), 3126-3133.

15. Petros, R. A.; DeSimone, J. M., Strategies in the design of nanoparticles for therapeutic applications. Nature reviews Drug discovery 2010, 9 (8), 615-627.

16. Hu, Y.; Xie, J.; Tong, Y. W.; Wang, C.-H., Effect of PEG conformation and particle size on the cellular uptake efficiency of nanoparticles with the HepG2 cells. Journal of Controlled Release 2007, 118 (1), 7-17.

17. Kumari, A.; Yadav, S. K.; Yadav, S. C., Biodegradable polymeric nanoparticles based drug delivery systems. Colloids and Surfaces B: Biointerfaces 2010, 75 (1), 1-18.

18. Hamblett, K. J.; Senter, P. D.; Chace, D. F.; Sun, M. M.; Lenox, J.; Cerveny, C. G.; Kissler, K. M.; Bernhardt, S. X.; Kopcha, A. K.; Zabinski, R. F., Effects of drug loading on the antitumor activity of a monoclonal antibody drug conjugate. Clinical Cancer Research 2004, 10 (20), 7063-7070.

19. Alonso, M. J.; Losa, C.; Calvo, P.; Vila-Jato, J., Approaches to improve the association of amikacin sulphate to poly (alkylcyanoacrylate) nanoparticles. International journal of pharmaceutics 1991, 68 (1-3), 69-76.

20. Paliwal, R.; Babu, R. J.; Palakurthi, S., Nanomedicine scale-up technologies: feasibilities and challenges. AAPS PharmSciTech 2014, 15 (6), 1527-1534.

21. Rabanel, J. M.; Latreille, P. L.; Lalloz, A.; Hildgen, P.; Banquy, X., Chapter 4 - Nanostructured nanoparticles for improved drug delivery A2 - Andronescu, Ecaterina. In Nanostructures for Drug Delivery, Grumezescu, A. M., Ed. Elsevier: 2017; pp 149-182.

22. Vauthier, C.; Bouchemal, K., Methods for the preparation and manufacture of polymeric nanoparticles. Pharmaceutical research 2009, 26 (5), 1025-1058.

23. Allouche, J., Synthesis of organic and bioorganic nanoparticles: an overview of the preparation methods. In Nanomaterials: A Danger or a Promise?, Springer: 2013; pp 27-74.

24. Lepeltier, E.; Bourgaux, C.; Couvreur, P., Nanoprecipitation and the "Ouzo effect": Application to drug delivery devices. Adv Drug Deliver Rev 2014, 71, 86-97.

25. Miladi, K.; Sfar, S.; Fessi, H.; Elaissari, A., Nanoprecipitation Process: From Particle Preparation to In Vivo Applications. In Polymer Nanoparticles for Nanomedicines, Springer: 2016; pp 17-53.

26. Pustulka, K. M.; Wohl, A. R.; Lee, H. S.; Michel, A. R.; Han, J.; Hoye, T. R.; McCormick, A. V.; Panyam, J.; Macosko, C. W., Flash nanoprecipitation: particle structure and stability. Molecular pharmaceutics 2013, 10 (11), 4367-4377.

27. Johnson, B. K.; Prud'homme, R. K., Flash nanoprecipitation of organic actives and block copolymers using a confined impinging jets mixer. Australian Journal of Chemistry 2003, 56 (10), 1021-1024.

28. Karnik, R.; Gu, F.; Basto, P.; Cannizzaro, C.; Dean, L.; Kyei-Manu, W.; Langer, R.; Farokhzad, O. C., Microfluidic Platform for Controlled Synthesis of Polymeric Nanoparticles. Nano Letters 2008, 8 (9), 2906-2912.

29. Stroock, A. D.; Dertinger, S. K. W.; Ajdari, A.; Mezić, I.; Stone, H. A.; Whitesides, G. M., Chaotic Mixer for Microchannels. Science 2002, 295 (5555), 647-651.

30. Kee, S. P.; Gavriilidis, A., Design and characterisation of the staggered herringbone mixer. Chem Eng J 2008, 142 (1), 109-121.

31. Quintanar-Guerrero, D.; Allémann, E.; Doelker, E.; Fessi, H., A mechanistic study of the formation of polymer nanoparticles by the emulsification-diffusion technique. Colloid and Polymer Science 1997, 275 (7), 640-647. 
32. Mora-Huertas, C. E.; Fessi, H.; Elaissari, A., Influence of process and formulation parameters on the formation of submicron particles by solvent displacement and emulsificationdiffusion methods. Advances in Colloid and Interface Science 2011, 163 (2), 90-122.

33. Avdeef, A., Absorption and drug development: solubility, permeability, and charge state. John Wiley \& Sons: 2012.

34. Avdeef, A.; Berger, C. M.; Brownell, C., pH-metric solubility. 2: Correlation between the acid-base titration and the saturation shake-flask solubility-pH methods. Pharmaceut Res 2000, 17 (1), 85-89.

35. Loftsson, T.; Hreinsdóttir, D.; Másson, M., The complexation efficiency. Journal of Inclusion Phenomena and Macrocyclic Chemistry 2007, 57 (1-4), 545-552.

36. Bergström, C. A.; Strafford, M.; Lazorova, L.; Avdeef, A.; Luthman, K.; Artursson, P., Absorption classification of oral drugs based on molecular surface properties. Journal of medicinal chemistry 2003, 46 (4), 558-570.

37. O'Neil, M. J., The Merck index: an encyclopedia of chemicals, drugs, and biologicals. RSC Publishing: 2013.

38. Kim, S.; Thiessen, P. A.; Bolton, E. E.; Chen, J.; Fu, G.; Gindulyte, A.; Han, L.; He, J.; He, S.; Shoemaker, B. A., PubChem substance and compound databases. Nucleic acids research 2015, 44 (D1), D1202-D1213.

39. Essa, S.; Rabanel, J. M.; Hildgen, P., Effect of polyethylene glycol (PEG) chain organization on the physicochemical properties of poly (D, L-lactide)(PLA) based nanoparticles. European journal of pharmaceutics and biopharmaceutics 2010, 75 (2), 96-106.

40. Nadeau, V.; Leclair, G.; Sant, S.; Rabanel, J.-M.; Quesnel, R.; Hildgen, P., Synthesis of new versatile functionalized polyesters for biomedical applications. Polymer 2005, 46 (25), 11263 11272.

41. Han, J.; Zhu, Z.; Qian, H.; Wohl, A. R.; Beaman, C. J.; Hoye, T. R.; Macosko, C. W., A simple confined impingement jets mixer for flash nanoprecipitation. Journal of Pharmaceutical Sciences 2012, 101 (10), 4018-4023.

42. Marrache, S.; Dhar, S., Engineering of blended nanoparticle platform for delivery of mitochondria-acting therapeutics. Proceedings of the National Academy of Sciences 2012, 109 (40), 16288-16293.

43. Lebouille, J. G. J. L.; Stepanyan, R.; Slot, J. J. M.; Stuart, M. A. C.; Tuinier, R., Nanoprecipitation of polymers in a bad solvent. Colloid Surface $A$ 2014, 460, 225-235.

44. Stepanyan, R.; Lebouille, J. G. J. L.; Slot, J. J. M.; Tuinier, R.; Stuart, M. A. C., Controlled Nanoparticle Formation by Diffusion Limited Coalescence. Phys Rev Lett 2012, 109 (13).

45. Johnson, B. K.; Prud'homme, R. K., Chemical processing and micromixing in confined impinging jets. Aiche J 2003, 49 (9), 2264-2282.

46. Le Dévédec, F.; Her, S.; Vogtt, K.; Won, A.; Li, X.; Beaucage, G.; Yip, C.; Allen, C., Drug governs the morphology of polyalkylated block copolymer aggregates. Nanoscale 2017, 9 (7), 2417-2423.

47. Rabanel, J.-M.; Faivre, J.; Paka, G. D.; Ramassamy, C.; Hildgen, P.; Banquy, X., Effect of polymer architecture on curcumin encapsulation and release from PEGylated polymer nanoparticles: Toward a drug delivery nano-platform to the CNS. European Journal of Pharmaceutics and Biopharmaceutics 2015, 96, 409-420.

48. Sant, S.; Nadeau, V.; Hildgen, P., Effect of porosity on the release kinetics of propafenoneloaded PEG-g-PLA nanoparticles. J Control Release 2005, 107 (2), 203-14.

49. Peer, D.; Karp, J. M.; Hong, S.; Farokhzad, O. C.; Margalit, R.; Langer, R., Nanocarriers as an emerging platform for cancer therapy. Nat Nano 2007, 2 (12), 751-760. 
1 50. Barichello, J. M.; Morishita, M.; Takayama, K.; Nagai, T., Encapsulation of hydrophilic and 2 lipophilic drugs in PLGA nanoparticles by the nanoprecipitation method. Drug development and 3 industrial pharmacy 1999, 25 (4), 471-476.

$451 . \quad$ Kumar, V.; Prud'Homme, R. K., Thermodynamic Limits on Drug Loading in Nanoparticle 5 Cores. J Pharm Sci 2008, 97 (11), 4904-4914.

6 


\title{
SUPPORTING INFORMATION
}

\section{Unified scaling of the structure and loading of nanoparticles formed via diffusion-limited coalescence}

\author{
Teresita Rode García ${ }^{1}$, Araceli García Ac ${ }^{1}$, Augustine Lalloz ${ }^{1}$, Francois-Xavier Lacasse ${ }^{1}$, \\ Patrice Hildgen ${ }^{1}$, Jean-Michel Rabanel ${ }^{1, \$,}$, Xavier Banquy ${ }^{1, *}$ \\ ${ }^{1}$ Faculté de Pharmacie, Université de Montréal, C.P. 6128, Succursale Centre-ville, \\ Montréal, Québec H3C 3J7, Canada. \\ ${ }^{\$}$ Present address: Centre INRS Institut Armand-Frappier, 531 Boulevard des Prairies, \\ Laval, Québec H7V 1B7, Canada \\ *Corresponding authors: xavier.banquy@umontreal.ca; jean-michel.rabanel@iaf.inrs.ca
}

\section{Table of content}

1. Polymer characterization: ${ }^{1} \mathrm{H}-\mathrm{NMR}$

2. NP fabrication methods approach

3. Effect of the fabrication method on the size PdI

4. Effect of D:P on PEG surface coverage

5. PEG chains organization according to the surface PEG density

6. Parameters used in Eq. 4

7. Zeta potential and stability of NPs.

8. References 


\section{Polymer characterisation: ${ }^{1}$ H-NMR}

${ }^{1} \mathrm{H}$ NMR spectrum (Figure S1) displays a characteristic peak at $5.2 \mathrm{ppm}$ corresponding to the tertiary PLA proton $(\mathrm{m},-\mathrm{CH})$, a peak at $3.6 \mathrm{ppm}$ for the protons of the repeating units on the PEG chain $\left(\mathrm{m}, \mathrm{OCH}_{2}-\mathrm{CH}_{2} \mathrm{O}\right)$, a peak at $3.3 \mathrm{ppm}$ for the pendant methyl group of the PEG chain $\left(\mathrm{m}-\mathrm{CH}_{3}\right)$ and a peak at $1.5 \mathrm{ppm}$ for the pendant methyl group of the repeating units on the PLA chain (m$\mathrm{CH}_{3}$ ). The composition of the synthesized diblock was calculated using the peak intensity ratio of PEG (3.6 ppm) to that PLA (5.2 ppm).

For this study, the diblock polymer was synthesized by ROP using Methoxy-PEG 2kDa as the chain initiator. In the literature, several synthesis strategies have been proposed to produce PEG-polyester diblocks. One of the most reported strategy relies on a two steps procedure, in which the polyester chain (such as PLA) is first synthetized (by condensation-polymerization or ring opening), followed by a coupling reaction to graft the PEG chain to the polyester. Nevertheless, in this type of reaction, the conjugation efficiency is very low, typically 30 to $40 \%{ }^{1}$ in contrast with the strategy chosen for this study yielding a $>90 \%$ of chains with a PEG segment attached as assessed by NMR.
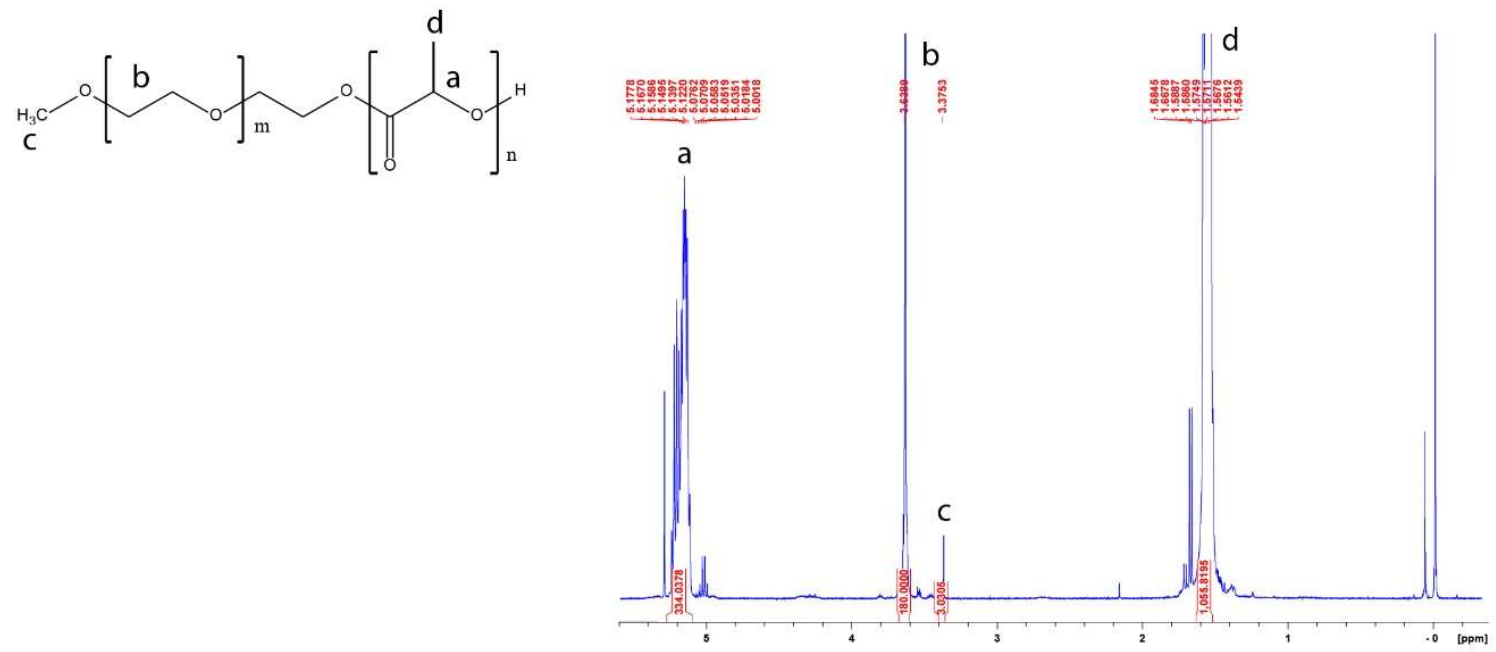

Figure S1. ${ }^{1} \mathrm{H}-\mathrm{MNR}$ spectra and chemical structure of PEG- $b$-PLA. 


\section{NP fabrication methods}

The NPs were produced by three different methods using mixing chambers of different geometries (see Figure 1A). For Batch Nanoprecipitation (BN), the mixing process takes place in a glass beaker $(100 \mathrm{~mL}$ ), the organic phase (which contains the polymer and the drug) being injected into the aqueous phase under stirring. When the semi-polar solvent mixes with the water, the waterinsoluble polymer precipitates to form the NPs. The other two methods used significantly smaller mixing chambers ( 3 orders of magnitude smaller) in which continuous precipitation occurs (Continuous Flow Nanoprecipitation process or CFN). The first method we tested was Flash Nanoprecipitation, which uses a confined mixer (Confined Impinging Jet Micromixer or CIJM) where the organic and aqueous phases are injected and collided in the center of a cylindrical mixing chamber. The collision of the two phases results in a fast mixing process and the NPs form continuously until reaching the storage reservoir. The second CFN process tested was a microfluidic-based Staggered Herringbone Micromixer (SHM). The mixing process of this micromixer relies on the strong transverse flow in the channel caused by the grooves geometry. The vertices of the grooves are offset to approximately one third of the way across the channel width, and each set of herringbones alternates with a complementary set to create a full mixing cycle. This alternation reorients the flow periodically producing chaotic mixing. ${ }^{2}$

Acetone was selected as the organic phase since it is less toxic (classified as a Class 3 solvent

according the US Pharmacopoeia ${ }^{3}$ ) than other solvents commonly used such as tetrahydrofuran or acetonitrile (which are Class 2 solvents). In addition, its low boiling point allows it to be easily removed by evaporation. Additionally, it has been reported that acetone allows higher fabrication yields compared to other water-soluble organic solvent ${ }^{4}$.

After fabrication, the nanosuspensions were dialysed to remove the remaining organic solvent and the non-encapsulated drug (see Fig.1 for the different purification methods associated to each property measured). The dialysis step was necessary to prevent particle aggregation ${ }^{5}$. 
Table S1. Composition of the organic phase, Polymer amount was kept constant while amount of drug was varied.

\begin{tabular}{|c|c|c|c|}
\hline $\begin{array}{c}\text { Drug : polymer } \\
\text { Ratio } \\
\%\end{array}$ & $\begin{array}{l}\text { Amount of } \\
\text { polymer } \\
m g\end{array}$ & $\begin{array}{c}\text { Amount of drug } \\
m g\end{array}$ & $\begin{array}{c}\text { Vol. of acetone } \\
\qquad m L\end{array}$ \\
\hline (Blank NPs) & 60 & 0 & 5 \\
\hline 1 & 60 & 0.6 & 5 \\
\hline 5 & 60 & 3.0 & 5 \\
\hline 10 & 60 & 6.0 & 5 \\
\hline 15 & 60 & 9.0 & 5 \\
\hline 20 & 60 & 12.0 & 5 \\
\hline
\end{tabular}

\section{Effect of the fabrication method on the PdI}

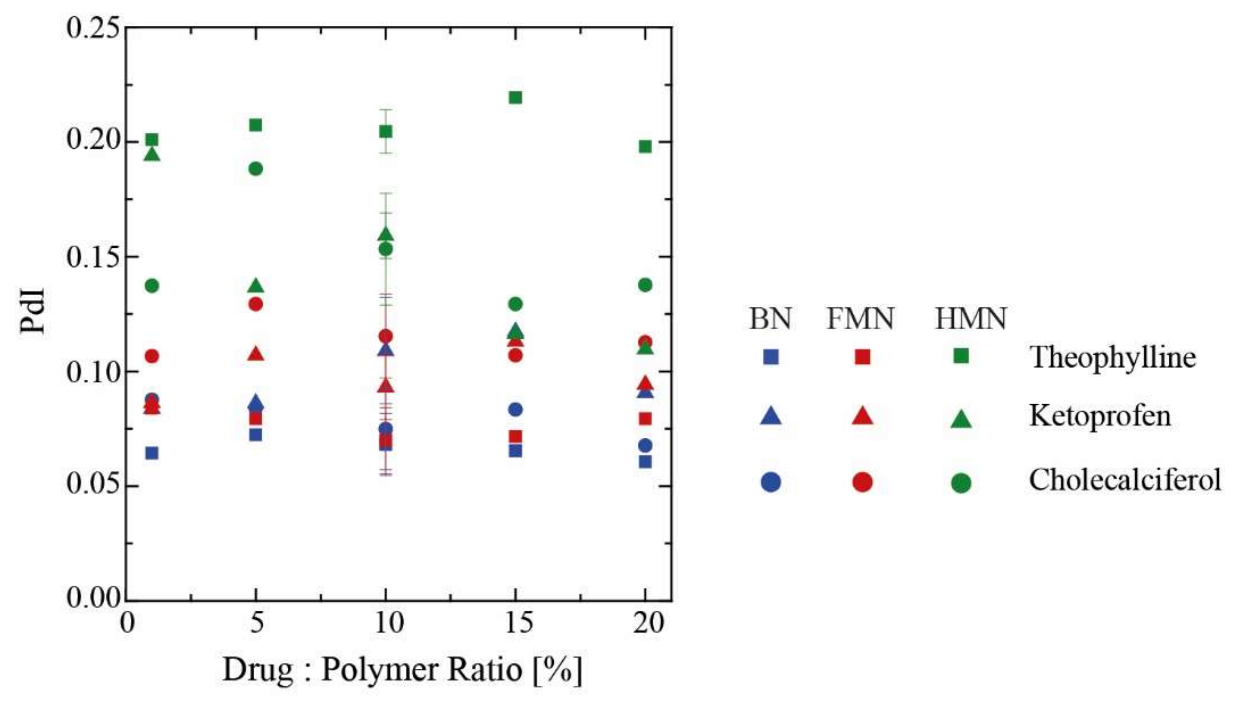

Figure S2. Effect of the fabrication method and amount of drug in the formulation (D:P) on the size PdI. 


\section{Effect of D:P on PEG surface coverage}
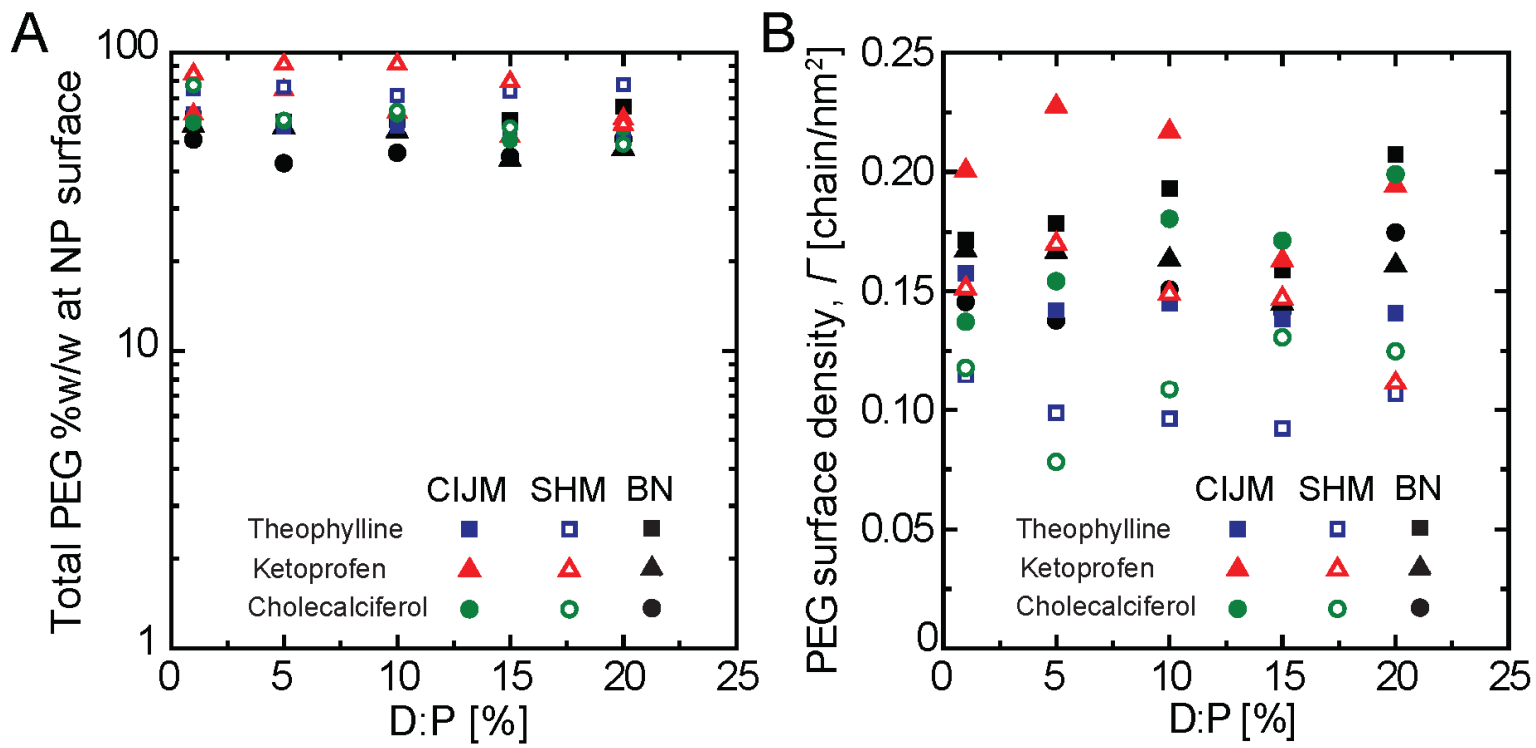

Figure S3. Distribution of PEG in the NP. (A) PEG content at the NP surface according to the drug : polymer ratio. (B) PEG density at the NP surface according to drug : polymer ratio.

\section{PEG chains organization according to the surface PEG density}

Table S2. Determination of arrangement of PEG chains at the surface of the NP.

\begin{tabular}{|c|c|c|c|c|c|}
\hline Drug & $\begin{array}{c}\text { Fabrication } \\
\text { Method }\end{array}$ & $\begin{array}{l}\text { Average PEG } \\
\text { density } \\
\text { (chains } / \mathrm{nm}^{2} \text { ) }\end{array}$ & $\begin{array}{c}\text { Distance } \\
\text { between PEG } \\
\text { chains (D) } \\
\text { (nm) }\end{array}$ & $\begin{array}{c}\text { Relation } \\
\text { D vs } \\
\mathrm{R}_{\mathrm{F}}^{*}\end{array}$ & $\begin{array}{l}\text { PEG corona } \\
\text { organization }\end{array}$ \\
\hline \multirow{3}{*}{ Theophylline } & $\mathrm{BN}$ & $0.193 \pm 0.016$ & 2.277 & $\mathrm{D}<\mathrm{RF}$ & Dense brush \\
\hline & CIJM & $0.145 \pm 0.015$ & 2.627 & $\mathrm{D}<\mathrm{RF}$ & Dense brush \\
\hline & SHM & $0.096 \pm 0.017$ & 3.227 & $\mathrm{D}<\mathrm{RF}$ & Dense brush \\
\hline \multirow{3}{*}{ Ketoprofen } & $\mathrm{BN}$ & $0.163 \pm 0.042$ & 2.474 & $\mathrm{D}<\mathrm{RF}$ & Dense brush \\
\hline & CIJM & $0.217 \pm 0.034$ & 2.147 & $\mathrm{D}<\mathrm{RF}$ & Dense brush \\
\hline & SHM & $0.149 \pm 0.025$ & 2.589 & $\mathrm{D}<\mathrm{RF}$ & Dense brush \\
\hline \multirow{3}{*}{ Cholecalciferol } & $\mathrm{BN}$ & $0.151 \pm 0.006$ & 2.576 & $\mathrm{D}<\mathrm{RF}$ & Dense brush \\
\hline & CIJM & $0.180 \pm 0.016$ & 2.355 & $\mathrm{D}<\mathrm{RF}$ & Dense brush \\
\hline & SHM & $0.109 \pm 0.032$ & 3.035 & $\mathrm{D}<\mathrm{RF}$ & Dense brush \\
\hline
\end{tabular}

$* \mathrm{R}_{\mathrm{F}}$ of PEG $2000=3.4 \mathrm{~nm}$ 


\section{Parameters used in Eq. 4}

\section{PEG}

Molar mass: $2000 \mathrm{~g} / \mathrm{mol}$

Molar volume $\mathrm{Vm}=1769.91 \mathrm{~mL} \mathrm{~mol}^{-1}$

Kuhn monomer length $\mathrm{b}=11 \AA$

Density: $1.13 \mathrm{~g} \cdot \mathrm{cm}^{-3}$

Total solubility parameter $\delta_{\mathrm{t}}: 22.2 \mathrm{MPa}^{1 / 2}$

PLA

Molar mass: $24000 \mathrm{~g} / \mathrm{mol}$

Molar volume $\mathrm{Vm}=19047.61 \mathrm{~mL} \mathrm{~mol}^{-1}$

Density $1.26 \mathrm{~g} \cdot \mathrm{cm}^{-3}$

Total solubility parameter $\delta_{\mathrm{t}}: 20.2 \mathrm{MPa}^{1 / 2}$

PLA-b-PEG

Interfacial tension, $\gamma_{\text {eff: }}: 23 \mathrm{mN} . \mathrm{m}$

Flory-Huggins interaction parameter, $\chi$, was obtained using the equation:

$$
\chi_{12}=\frac{\left(\delta_{1}-\delta_{2}\right)^{2} V_{m 1}}{R T}
$$

where 1 is the small molecule (drug)

The calculated values of the Drug/PLA and Drug/PEG interaction parameters are:

$\chi_{12}$ drug/PLA

Theophylline

Ketoprofen

Cholecalcipherole

0.23

$\chi_{12}$ Theophylline/PEG

2.12 


\section{Zeta potential and stability of NPs.}

Table S3. Zeta potential of NP's prepared by different methods. Data collected at D:P $=10 \% \mathrm{w} / \mathrm{w}$

\begin{tabular}{c|c|c}
\hline Drug & $\begin{array}{c}\text { Fabrication } \\
\text { Method }\end{array}$ & $\begin{array}{c}\text { Zeta Potential } \\
(\mathrm{mV})\end{array}$ \\
\hline \multirow{3}{*}{ Theophylline } & $\mathrm{BN}$ & $-23.83 \pm 0.76$ \\
& CIJM & $-19.57 \pm 2.10$ \\
& SHM & $-23.38 \pm 1.87$ \\
\hline & & \\
\hline \multirow{3}{*}{ Ketoprofen } & BN & $-12.47 \pm 0.85$ \\
& CIJM & $-13.22 \pm 3.16$ \\
& SHM & $-10.93 \pm 0.73$ \\
\hline \multirow{3}{*}{ Cholecalciferol } & & \\
& CIJM & $-22.72 \pm 0.56$ \\
& SHM & $-23.35 \pm 1.23$ \\
\hline
\end{tabular}

Table S4. NP's size monitored over 7 days. Data collected at D:P $=10 \% \mathrm{w} / \mathrm{w}$

\begin{tabular}{c|c|c|c|c}
\hline \multirow{2}{*}{ Drug } & \multirow{2}{*}{$\begin{array}{c}\text { Fabrication } \\
\text { Method }\end{array}$} & \multicolumn{3}{|c}{$\begin{array}{c}\text { Hydrodynamic diameter, } \mathrm{D}_{\mathrm{H}} \\
(\mathrm{nm})\end{array}$} \\
\cline { 3 - 5 } & & Day 0 & Day 3 & Day 7 \\
\cline { 3 - 5 } Theophylline & BN & $101.68 \pm 5.34$ & $104.03 \pm 1.10$ & $104.18 \pm 1.94$ \\
& CIJM & $86.00 \pm 0.60$ & $85.92 \pm 0.66$ & $88.38 \pm 1.07$ \\
& SHM & $63.70 \pm 5.03$ & $61.84 \pm 0.19$ & $64.76 \pm 5.95$ \\
\hline & & & & \\
\hline \multirow{3}{*}{ Ketoprofen } & BN & $117.99 \pm 3.22$ & $119.11 \pm 1.75$ & $120.88 \pm 1.64$ \\
& CIJM & $108.18 \pm 0.89$ & $103.93 \pm 1.10$ & $105.49 \pm 3.39$ \\
& SHM & $71.77 \pm 2.25$ & $76.04 \pm 1.56$ & $75.20 \pm 5.37$ \\
\hline \multirow{3}{*}{ Cholecalciferol } & & & & \\
& BN & $119.26 \pm 1.41$ & $117.97 \pm 1.31$ & $119.72 \pm 2.82$ \\
& SHM & $122.98 \pm 4.37$ & $124.55 \pm 3.84$ & $118.11 \pm 2.39$ \\
& & $107.12 \pm 3.16$ & $106.50 \pm 0.67$ & $102.07 \pm 0.94$ \\
\hline
\end{tabular}


Table S5. NP's zeta potential monitored over 7 days. Data collected at D:P $=10 \% \mathrm{w} / \mathrm{w}$

\begin{tabular}{c|c|c|c|c}
\hline \multirow{2}{*}{ Drug } & \multirow{2}{*}{$\begin{array}{c}\text { Fabrication } \\
\text { Method }\end{array}$} & \multicolumn{3}{|c}{$\begin{array}{c}\text { Zeta Potential } \\
(\mathrm{mV})\end{array}$} \\
\cline { 3 - 5 } & & Day 0 & Day 3 & Day 7 \\
\cline { 3 - 5 } Theophylline & BN & $-23.83 \pm 0.76$ & $-23.07 \pm 0.29$ & $-19.77 \pm 2.54$ \\
& CIJM & $-19.57 \pm 2.10$ & $-21.70 \pm 2.08$ & $-20.52 \pm 2.04$ \\
& SHM & $-23.38 \pm 1.87$ & $-23.53 \pm 1.18$ & $-22.53 \pm 4.41$ \\
\hline & & & & \\
\hline \multirow{3}{*}{ Ketoprofen } & BN & $-12.47 \pm 0.85$ & $-14.48 \pm 0.85$ & $-15.48 \pm 3.96$ \\
& CIJM & $-13.22 \pm 3.16$ & $-12.92 \pm 1.65$ & $-13.10 \pm 0.65$ \\
& SHM & $-10.93 \pm 0.73$ & $-10.83 \pm 2.12$ & $-10.98 \pm 2.15$ \\
\hline \multirow{3}{*}{ Cholecalciferol } & & & & \\
& BN & $-22.72 \pm 0.56$ & $-22.02 \pm 0.25$ & $-22.20 \pm 2.17$ \\
& SHM & $-22.79 \pm 1.11$ & $-20.77 \pm 1.73$ & $-22.26 \pm 4.74$ \\
\hline
\end{tabular}

\section{References}

1. Marrache, S.; Dhar, S., Engineering of blended nanoparticle platform for delivery of mitochondria-acting therapeutics. Proceedings of the National Academy of Sciences 2012, 109 (40), 16288-16293.

2. Stroock, A. D.; Dertinger, S. K. W.; Ajdari, A.; Mezić, I.; Stone, H. A.; Whitesides, G. M., Chaotic Mixer for Microchannels. Science 2002, 295 (5555), 647-651.

3. United States Pharmocopeial Convention, I., United States Pharmacopeia 35, National Formulary 30. In 476, Residual Solvents, 35 ed.; Rockville, MD, 2012; Vol. 1, p 200.

4. Legrand, P.; Lesieur, S.; Bochot, A.; Gref, R.; Raatjes, W.; Barratt, G.; Vauthier, C., Influence of polymer behaviour in organic solution on the production of polylactide nanoparticles by nanoprecipitation. International Journal of Pharmaceutics 2007, 344 (1), 33-43.

5. Saad, W. S.; Prud'homme, R. K., Principles of nanoparticle formation by flash nanoprecipitation. Nano Today 2016, 11 (2), 212-227. 